\title{
Reorganization of gene network for degradation of polycyclic aromatic hydrocarbons (PAHs) in Pseudomonas aeruginosa PAO1 under several conditions
}

\author{
Shaomin Yan $^{1} \cdot$ Guang $\mathrm{Wu}^{1}$ (i)
}

Received: 28 November 2016/Revised: 22 May 2017 / Accepted: 6 June 2017 /Published online: 7 July 2017

(C) The Author(s) 2017. This article is an open access publication

\begin{abstract}
Although polycyclic aromatic hydrocarbons (PAHs) are harmful to human health, their elimination from the environment is not easy. Biodegradation of PAHs is promising since many bacteria have the ability to use hydrocarbons as their sole carbon and energy sources for growth. Of various microorganisms that can degrade PAHs, Pseudomonas aeruginosa is particularly important, not only because it causes a series of diseases including infection in cystic fibrosis patients, but also because it is a model bacterium in various studies. The genes that are responsible for degrading PAHs have been identified in $P$. aeruginosa, however, no gene acts alone as various stresses often initiate different metabolic pathways, quorum sensing, biofilm formation, antibiotic tolerance, etc. Therefore, it is important to study how PAH degradation genes behave under different conditions. In this study, we apply network analysis to investigating how 46 PAH degradation genes reorganized among 5549 genes in $P$. aeruginosa $\mathrm{PAO} 1$ under nine different conditions using publicly available gene coexpression data from GEO. The results provide six aspects of novelties: (i) comparing the number of gene clusters before and after stresses, (ii) comparing the membership in each gene cluster before and after stresses, (iii) defining which gene changed its membership together with PAH degradation genes before and after stresses,
\end{abstract}

Communicated by: Agnieszka Szalewska-Palasz

Electronic supplementary material The online version of this article (doi:10.1007/s13353-017-0402-9) contains supplementary material, which is available to authorized users.

Guang Wu

hongguanglishibahao@yahoo.com

1 Bioscience and Technology Research Center, Guangxi Academy of Sciences, 98 Daling Road, Nanning, Guangxi 530007, China (iv) classifying membership-changed-genes in terms of category in Pseudomonas Genome Database, (v) postulating unknown gene's function, and (vi) proposing new mechanisms for genes of interests. This study can shed light on understanding of cooperative mechanisms of PAH degradation from the level of entire genes in an organism, and paves the way to conduct the similar studies on other genes.

Keywords Bioinformatics $\cdot$ Network $\cdot$ Polycyclic aromatic hydrocarbon degradation gene $\cdot$ Pseudomonas Aeruginosa

\section{Introduction}

Hydrocarbons are major components of crude oil, but they are harmful to human health, especially polycyclic aromatic hydrocarbons (PAHs), which are mainly generated by incomplete combustion (Yan et al. 2004). PAHs exist not only in fossil fuels but also in biomass because the complex aromatic polymer lignin accounts for a quarter of the land-based biomass on Earth (Kirk and Farrell 1987), therefore the burning of sugarcane also generates PAHs (Ferreira et al. 2014). More recently, it is reported that PAHs take part in the formation of urban haze (Guo et al. 2014).

Great efforts are made to eliminate PAHs from the environment through physical, chemical, and biological approaches (Floehr et al. 2013). Biodegradation of PAHs by microorganisms is promising (Peng et al. 2008; Kanaly and Harayama 2000) because many bacteria have the ability to use hydrocarbons as their sole carbon and energy sources for growth, e.g., Alcaligenes faecalis AFK2 (Kiyohara et al. 1982), Arthrobacter sp. P1-1 (Seo et al. 2006), Burkholderia sp. RP007 and C3 (Laurie and Lloyd-Jones 1999; Seo et al. 2007), Comamonas testeroni GZ42 (Goyal and Zylstra 1997), Mycobacterium vanbaalenii PYR-1 (Kweon et al. 
2014), Nocardioides $s p$. KP7 (Iwabuchi and Harayama 1998), Rhodococcus sp. NCIMB12038, P200 and P400 (Kulakov et al. 2000, 2005), Sphingobium yanoikuyae B1 (Zhao et al. 2015), and Terrabacter sp. DBF63 (Habe et al. 2003). Besides these bacteria, ligninolytic and nonligninolytic fungi, e.g., Phanerochaete chrysosporium (Bogan et al. 1996), Phanerochaete laevis (Bogan and Lamar 1996), and Bjerkandera adusta (Wang et al. 2003), are also able to mineralize PAHs (Cerniglia 1997).

PAHs can be classified as low-molecular weight and highmolecular weight PAHs, and their catabolism in bacteria is extremely diverse and complicated because of numerous PAHs, which numbered 922 not long ago (Sander and Wise 2011). In M. vanbaalenii PYR-1, for example, the degradation of pyrene needs 27 enzymes (Kim et al. 2007) whereas the degradation of fluoranthene requires 53 enzymes (Kweon et al. 2007). So far, many degradation pathways have been elucidated (Seo et al. 2007; Warhurst and Fewson 1994; Meckenstock et al. 2004), and their corresponding genes have been identified (Ghiorse et al. 1995; Xia et al. 2015). For instance, the aromatic ringcleaving dioxygenase opens the aromatic ring of single-ring aromatic compounds by incorporating two atoms of oxygen via the $\beta$-ketoapidate pathway (Frazee et al. 1993).

Pseudomonas aeruginosa is a Gram-negative bacterium and has an extremely important implication in a series of diseases, and currently serves as a model organism in quorum sensing studies (Lee and Zhang 2015). P. aeruginosa can decompose hydrocarbons (Patel et al. 2014; Liu et al. 2012), and lives in oil fields (Gai et al. 2012). In fact, many species of Pseudomonas have such ability, e.g., P. aeruginosa (Gai et al. 2012), P. alcaligenes PA-10 (Gordon and Dobson 2001), P. fluorescens ACB (Kamerbeek et al. 2001), P. putida NCIB 9816 (Yang et al. 1994), P. resinovorans (Nojiri et al. 2002), P. veronii (Onaca et al. 2007; Nam et al. 2003), and P. stutzeri (Bosch et al. 2000). As a matter of fact, P. paucimobilis EPA505 was isolated in 1990 because it uses fluoranthene as its sole source of carbon and energy (Mueller et al. 1990). Genetically, PAH degradation genes cluster together in Pseudomonas strains (Yang et al. 1994; Menn et al. 1993; Li et al. 2004; Balashova et al. 2001), whereas those genes scatter in Sphingomonads (Basta et al. 2005).

Interestingly, when pyrene was catalyzed in $M$. vanbaalenii PYR-1, as many as 142 proteins were upregulated although its degradation needs 27 enzymes (Kim et al. 2007). This not only indicates the existence of a complex network among genes, but also suggests that each gene dynamically and actively responds to different stresses. Indeed, different experimental conditions and different stresses should stimulate the genes to respond differently. Therefore, we are particularly interested in how PAH degradation genes in P. aeruginosa behave under different conditions with different stresses since this knowledge will render us a whole picture on how PAH degradation genes work.
Microarray analysis is certainly helpful because it simultaneously and globally examines the complete transcriptome in response to a stimulus by comparing mRNA levels before and after the stimulus, whose significance can be evaluated using statistical tests, absolute fold changes, and marginal call. In a broader sense, a stimulus not only regulates mRNA levels up or down, but also changes the relationship between any two gene expressions. The correlation between two genes indicates whether two genes act more coherently under the stimulus, but both genes do not necessarily have significance in up/down-regulation. Subsequently, the genes with high correlation are more likely to encode interacting proteins, or to have a similar biological function, or to belong to the same biological pathway (Braun 2014). Gene Expression Omnibus (GEO) (Edgar et al. 2002; Barrett et al. 2013) provides the opportunity to explore correlations between gene expressions. Doubtless, the study combining numerous coexpression data of $P$. aeruginosa from GEO is a big data study in accordance with BD2K (Big Data to Knowledge) proposed by National Institutes of Health (NIH) (Ohno-Machado 2014).

Hence, this study is designed to use network analysis with coexpression data of $P$. aeruginosa from GEO to investigate how PAH degradation genes behave under different circumstances with different stresses because technically network analysis can easily detect clusters; and thus, is an excellent tool to examine how PAHs genes work under different conditions with different stresses. In this context, network analysis produces the following novelties: (i) comparing the number of clusters before and after stresses, (ii) comparing the membership in each cluster before and after stresses, (iii) defining which gene changed its membership together with PAH degradation genes before and after stresses, (iv) classifying membershipchanged-genes in terms of category in Pseudomonas Genome Database, (v) postulating unknown gene's function, and (vi) proposing new mechanism on genes of interests.

\section{Materials and methods}

\section{Data}

Publicly available microarray data were obtained from GEO with raw datasets performed in platform GPL84, which is the [Pae_G1a] Affymetrix Pseudomonas aeruginosa Array (http:// www.affymetrix.com/support/technical/byproduct.affx?product= paeruginosa). This platform was used to study the transcriptional regulation and antimicrobial agent response of $P$. aeruginosa strain PAO1. GPL84 platform lists 5549 P. aeruginosa genes, which can be categorized according to the category in Pseudomonas Genome DataBase (Winsor et al. 2011, 2016).

The following stresses and conditions are included in this study: (i) the exposure of $P$. aeruginosa PAO1 to hydrogen peroxide because microorganisms continuously encounter 
various reactive oxygen species during their lifetime (Miller and Britigan 1997) and P. aeruginosa has a defense system against reactive oxidants (Ochsner et al. 2000), and 223 and 1854 genes were found statistically changed in coexpression experiments (accession number in GEO: GSE3090) (Chang et al. 2005; Palma et al. 2004); (ii) the exposure of $P$. aeruginosa PAO1 to sodium hypochlorite, which is the most widely used disinfectant, led to 457 and 625 genes upregulated and downregulated (accession number in GEO: GSE7402) (Small et al. 2007); (iii) the exposure of $P$. aeruginosa $\mathrm{PAO} 1$ to high and low oxygen concentrations because $P$. aeruginosa PAO1 can adapt to alternation of availability of oxygen in environment (accession number in GEO: GSE52445) (He et al. 2014); (iv) the exposure of P. aeruginosa PAO1 to ortho-phenylphenol because the exposure leads to transition to anaerobic respiration and swarming motility, and 509 genes were statistically found changed after exposure for 20 and $60 \mathrm{~min}$ (accession number in GEO: GSE10604) (Nde et al. 2008); (v) the expression of TpoN molecular roadblock in $P$. aeruginosa PAO1, which could potentially be used in any bacterium (accession number in GEO: GSE35632); (vi) subpopulations of biofilms of P. aeruginosa PAO1 because biofilms are spatially distinct and the top subpopulation is different from the bottom one (accession number in GEO: GSE34762) (Williamson et al. 2012); (vii) the exposure of $P$. aeruginosa PAO1 biofilms to ciprofloxacin and tobramycin because 340 and 683 genes were up- and down-regulated in the biofilm, 78 and 15 genes were up- and down-regulated with ciprofloxacin treatment, and 111 and 70 genes were up- and down-regulated with tobramycin treatment (accession number in GEO: GSE65882) (Stewart et al. 2015); (viii) the comparison of Australian epidemic strain-1 and 2 (P. aeruginosa AES-1 and AES-2) in planktonic culture and biofilm not only because these two strains prevail in Austrian cystic fibrosis patients (accession number in GEO: GSE6122 and GSE10304) (Manos et al. 2008, 2009) but also because their transcriptome analyses were done using $P$. aeruginosa PAO1 Affymetrix array; and (ix) the comparison between wild-type $P$. aeruginosa PAO1 and PA2449-null mutant $P$. aeruginosa PW5126 (accession number in GEO: GSE39044) (Lundgren et al. 2013) because phenazines, redox active compounds, are polyaromatic secondary metabolites controlled by PA2449.

\section{Genes for PAH degradation}

In the past, studies sometimes included alkane degradation genes, which are numbered 19 in P. aeruginosa PAO1, together with PAH degradation genes in analysis (Liu et al. 2012; Gai et al. 2012). However, this study excludes alkane degradation genes in order to get true insight into the network of PAH degradation genes under various conditions. Based on Kyoto Encyclopedia of Genes and Genomes (Kanehisa and
Goto 2000; Kanehisa et al. 2014, 2016), 46 PAH degradation genes were analyzed in this study as follows: PA0153, PA0154, PA0230, PA0231, PA0232, PA0247, PA0480, PA0817, PA0865, PA0880, PA1205, PA1210, PA1253, PA1966, PA2009, PA2024, PA2083, PA2085, PA2217, PA2418, PA2507, PA2508, PA2509, PA2512, PA2513, PA2514, PA2515, PA2516, PA2517, PA2518, PA2546, PA3240, PA3389, PA3629, PA3935, PA4091, PA4092, PA4121, PA4122, PA4123, PA4124, PA4125, PA4190, PA4486, PA4515, and PA5427.

\section{Gene network}

The genes with similar expression profiles are more likely (i) to encode interacting proteins, (ii) to have a similar biological function, and (iii) to belong to the same biological pathway (Braun 2014). In network analysis, a gene corresponds to a node and the correlation between any two gene expression profiles corresponds to an edge between two nodes, and a network can be thus constructed.

\section{Network analysis, heatmap and dendrogram}

Network analysis was conducted using iGraph R package (http://igraph.org/) and Pajek (de Nooy et al. 2011). Heatmap and dendrogram were constructed using R package.

\section{Results}

Figure 1 shows the network of 5548 genes from $P$. aeruginosa PAO1 including 46 PAH degradation genes before (panel A) and after exposure (panel B) to hydrogen peroxide. In these two gene networks, a symbol represents a gene with its locus tag, of which larger symbols represent PAH degradation genes. A line between two symbols indicates a good correlation between the coexpression profiles of these two genes. A cluster aggregates the symbols that more densely connect each other within their cluster but sparsely connect with the symbols in another cluster so the genes in a cluster are more likely to work together. Reorganization of genes is clearly visible in panel C, which can be read as follows, PA0153 together with 26 genes were found in a new cluster (Supplementary materials) according to the category in Pseudomonas Genome Database (Winsor et al. 2011, 2016).

Figure 2 displays the genes together with PAH degradation genes in new clusters after exposure to sodium hypochlorite. Actually, this study used the same control as panel A in Fig. 1 (Chang et al. 2005). Network analysis found that after the exposure to sodium hypochlorite the 5548 genes formed several huge clusters with the largest cluster containing 2047 genes, which is really different from the exposure to hydrogen peroxide in panel C in Fig. 1, where no cluster contains more 
than 200 genes. Thus, the number of genes associated with PAH degradation genes for regrouping in Fig. 2 is clearly higher than that in panel $\mathrm{C}$ in Fig. 1.

Figure 3 examines the gene networks of $P$. aeruginosa PAO1 after oxygen exposure from high to low oxygen concentration (upper panel) and from low to high oxygen concentration (lower panel) (He et al. 2014). At first glance, there is little difference between upper and lower panels, but the difference is that network finds 170 and 113 clusters for oxygen exposing from high to low oxygen concentration (upper panel) and from low to high oxygen concentration (lower panel). Cyan colored symbols at the bottom in both panels are 2334 and 2523 isolated genes for oxygen exposing from high to low oxygen concentration (upper panel) and from low to high oxygen concentration (lower panel), i.e., they have no connection with any other genes.

Figure 4 delineates the reorganizations of $P$. aeruginosa PAO1 genes after the exposure to ortho-phenylphenol for 20 and 60 min (Panels A and B) with respect to PAH degradation genes. For 5547 genes, network analysis discovers 18 clusters before the exposure and 15 and 16 clusters after the exposure for 20 and $60 \mathrm{~min}$, indicating that the genes did reorganize after the exposure. At $20 \mathrm{~min}$, unclassified genes, translation, ribosomal structure, and biogenesis genes and transcription genes reorganized strongly (last three rows along category axis in panel A), which is explainable because the initial response of $P$. aeruginosa PAO1 should increase its synthesis of defending protein. Two PAH degradation genes PA2514 and PA4123 associated with 121 genes at $20 \mathrm{~min}$ (panel A), among them 28 genes are associated with the transport and metabolism of amino acid or carbohydrate or inorganic ion. At 60 min, many genes are associated with PAH degradation gene PA5427 (last column along PAH degradation gene axis in panel B), including 38 genes having functions of translation, ribosomal structure, and biogenesis. Among them, PA4263 is a heat shock protein involved in stress response, and PA4266 is an elongation factor whose activity reflects a cellular protective response (Nde et al. 2008).

Figure 5 demonstrates the genes together with PAH degradation genes in new clusters before and after the expression of RpoN (PA4462) molecular roadblock. For 5549 genes, network analysis discovers 20 clusters before the expression and 19 clusters after the expression. As can be seen, RpoN roadblock did not lead many genes to regroup with PAH degradation genes compared with other figures. Actually, nine genes from PA2134 to PA2192 were found to have the relationship with PAH degradation genes, PA0154 vs PA2145, PA1253 vs PA2174, PA2508 vs PA2182, PA2514 vs PA2177, PA2516 vs PA2176, PA3240 vs PA2149, PA4121 vs PA2169, PA4122 vs PA2173, and PA5427 vs PA2143, whereas PA2173 and PA2176 are regulated by sigma factor $\sigma 22$ (AlgU, PA0762).

Figure 6 exhibits gene networks of the top $30 \mu \mathrm{m}$ (upper panel) and bottom $30 \mu \mathrm{m}$ (middle panel) of biofilm because P. aeruginosa PAO1 biofilms are spatially distinct and form
Fig. 1 Network of 5548 genes from P. aeruginosa PAO1 including 46 PAH degradation genes before (panel A) and after exposure (panel B) to hydrogen peroxide. Each symbol represents a gene with its code: circles represent non-PAH degradation genes and large triangles represent PAH degradation genes. Each edge represents a good correlation between two genes coexpression profiles. Because of space limitation, a cluster may not appear to contain many genes, but in reality, there are 521 genes in cyan cluster, 506 genes in yellow cluster, 398 genes in lime green cluster, 368 genes in red cluster, 361 genes in blue cluster, 356 genes in pink cluster, 343 genes in white cluster, 321 genes in orange cluster, 302 genes in purple cluster, 280 genes in cadet blue cluster, 253 genes in teal blue cluster, 252 genes in olive green cluster, 245 genes in gray cluster, 243 genes in black cluster, 225 genes in maroon cluster, 181 genes in light green cluster, 168 genes in light yellow cluster, 140 genes in magenta cluster, and 85 genes in midnight blue cluster in panel A. Panel C shows the genes together with PAH degradation genes found in new clusters after exposure to hydrogen peroxide in $P$. aeruginosa PAO1. Each pair of the following PAH degradation genes (PA0865 and PA4091, PA1205 and PA2217, PA2512 and PA4122, PA2515 and PA5427) have the same profiles

two subpopulations (Williamson et al. 2012). The top subpopulation, whose cell activities were high (Williamson et al. 2012), was composed of 89 clusters (upper panel), while the bottom subpopulation, whose cell activities were low (Williamson et al. 2012), was composed of 21 clusters (middle panel). The lower panel shows the percentage of the genes that changed their membership in five clusters, where each pie is a cluster in the bottom subpopulation of biofilms and its compositions are the genes belonging to the clusters in the top subpopulation of biofilms. For example, the first cluster in the bottom subpopulation (pie I in lower panel) is composed of $14.57 \%$ from cluster 0 of the top subpopulation, $17.46 \%$ from cluster 1 of the top subpopulation, and so on.

Figure 7 highlights the genes together with PAH degradation genes in new clusters after $12 \mathrm{~h}$ treatments of $P$. aeruginosa PAO1 biofilms with ciprofloxacin (left-hand panel) and tobramycin (right-hand panel) (Stewart et al. 2015). The first row in both panels shows the number of upregulated genes in biofilms with reference to PAH degradation genes. The second row in both panels shows the number of downregulated genes in biofilms with reference to PAH degradation genes. The third and fourth rows in both panels show the upregulated and downregulated genes with ciprofloxacin and tobramycin treatment, respectively.

Figure 8 illustrates the genes together with PAH degradation genes in new clusters of $P$. aeruginosa AES- 1 in biofilm (panel A) (Manos et al. 2008), and P. aeruginosa AES-2 in biofilm (panel B) (Manos et al. 2009). For 5549 genes, network analysis finds 16 and 18 clusters in AES-1 planktonic culture and biofilm, and 17 clusters in AES-2 planktonic culture and biofilm, suggesting biofilm influences AES-1 more than AES-2.

Figure 9 pictures gene networks of wild-type $P$. aeruginosa PAO1 and PA2449-null mutant $P$. aeruginosa PW5126 (Lundgren et al. 2013) because phenazines, redox active 
A

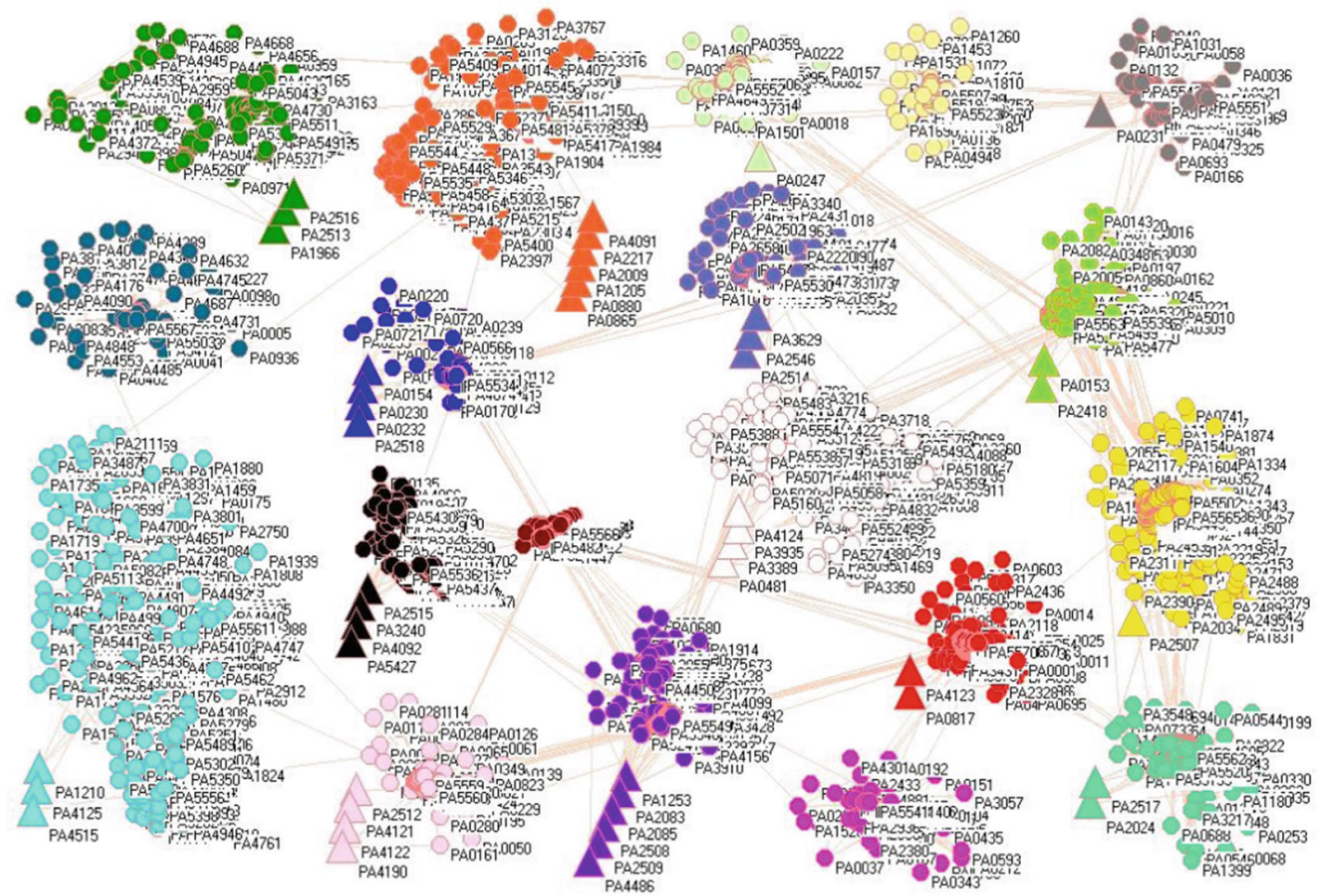

B

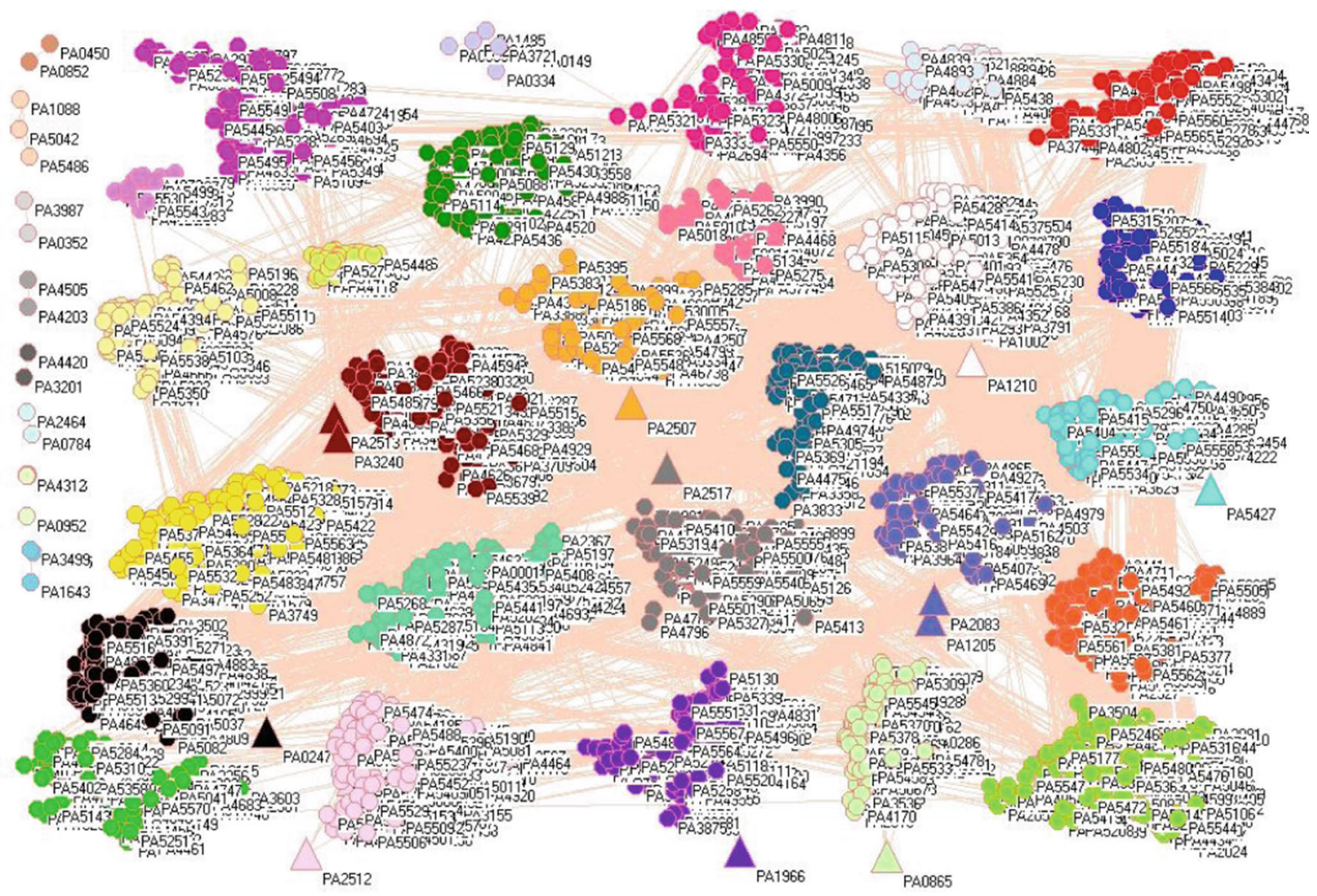

compounds, are polyaromatic secondary metabolites controlled by PA2449 and serve as virulence factors in
P. aeruginosa. Network analysis found 13 clusters in wildtype $P$. aeruginosa $\mathrm{PAO} 1$ (upper panel) but 14 clusters in 


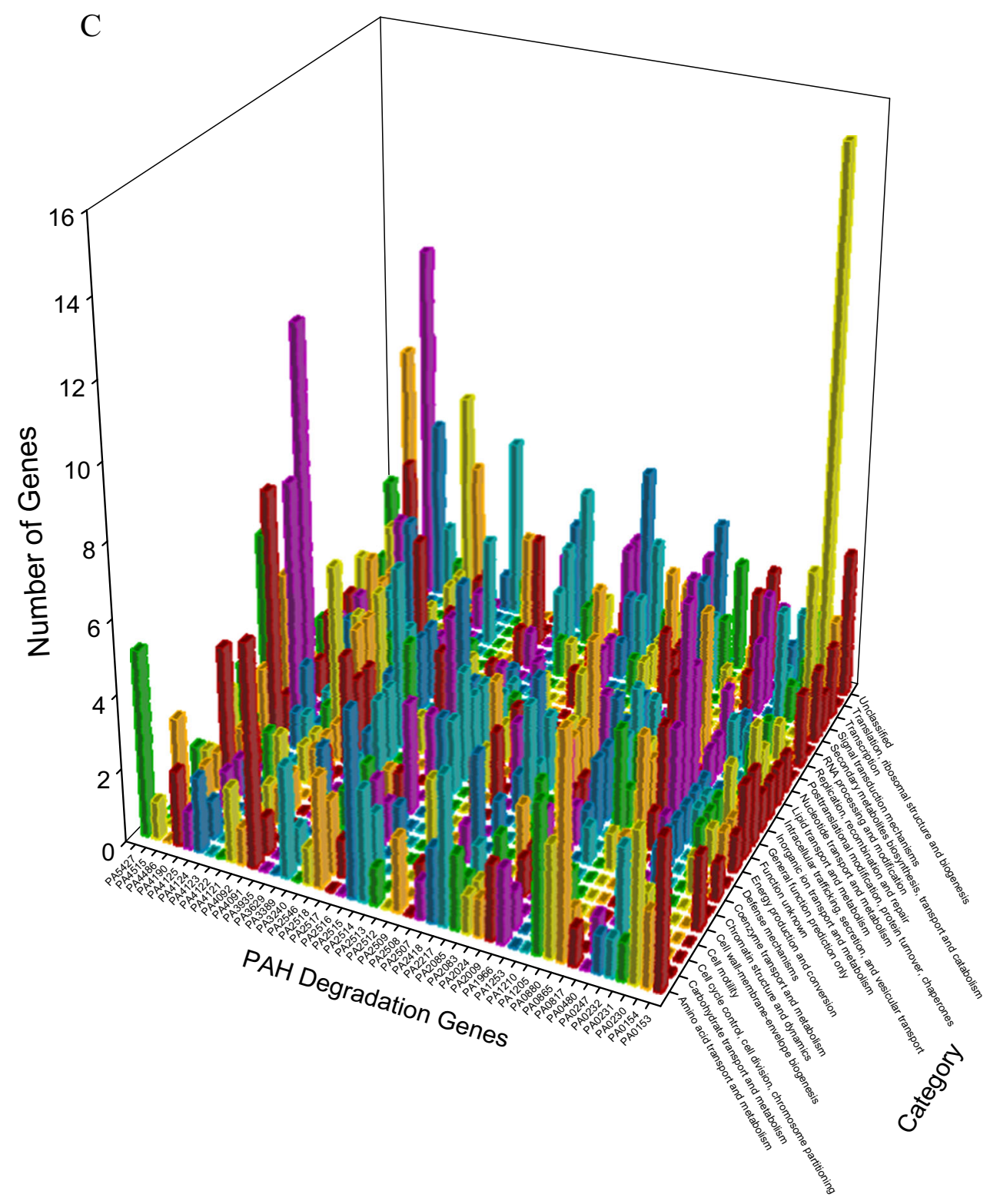

Fig. 1 (continued)

P. aeruginosa PW5126 (lower panel). Clearly, this difference is due to a mutation at PA2449.

Figure 10 summarizes how PAH-degradation genes reorganized with association of gene category in response to nine different stresses and conditions in terms of heatmap and cluster analysis. The color in heatmap begins from red to violet (red, orange, yellow, green, blue, indigo, and violet) representing the number of genes in terms of gene category increases from 0 to 112 , i.e., the bottom row has the highest number but two rows up to the bottom row have the lowest number. So the color indicates the degree of PAH degradation genes associated with different genes, which is in good agreement with the classification of the dendrogram on the lefthand side. With respect to columns, the dendrogram on the top depicts the hierarchical cluster tree of PAH degradation genes, from which we can trace different functions of $\mathrm{PAH}$ degradation genes.

\section{Discussion}

In this study, we attempted to use network analysis to uncover how PAH degradation genes work under nine different conditions. Ideally, it would be far better to have a PAH condition as a "positive control", unfortunately no such data are currently available. Moreover, the number of PAHs has already reached 922 (Sander and Wise 2011), and microarray on all PAHs would be very costly and time-consuming, but the microarray on a single PAH appears less attractive. In this situation, integrative analysis of available microarrays in different 
Fig. 2 Genes together with PAH degradation genes found in new clusters after exposure to sodium hypochlorite in $P$. aeruginosa PAO1. Each pair of the following PAH degradation genes have the same profiles: PA0230 and PA2518, PA0865 and PA0880, PA2083 and PA4486, PA2508 and PA2509, PA2514 and PA2546, PA3240 and PA4092, PA4121 and PA4122

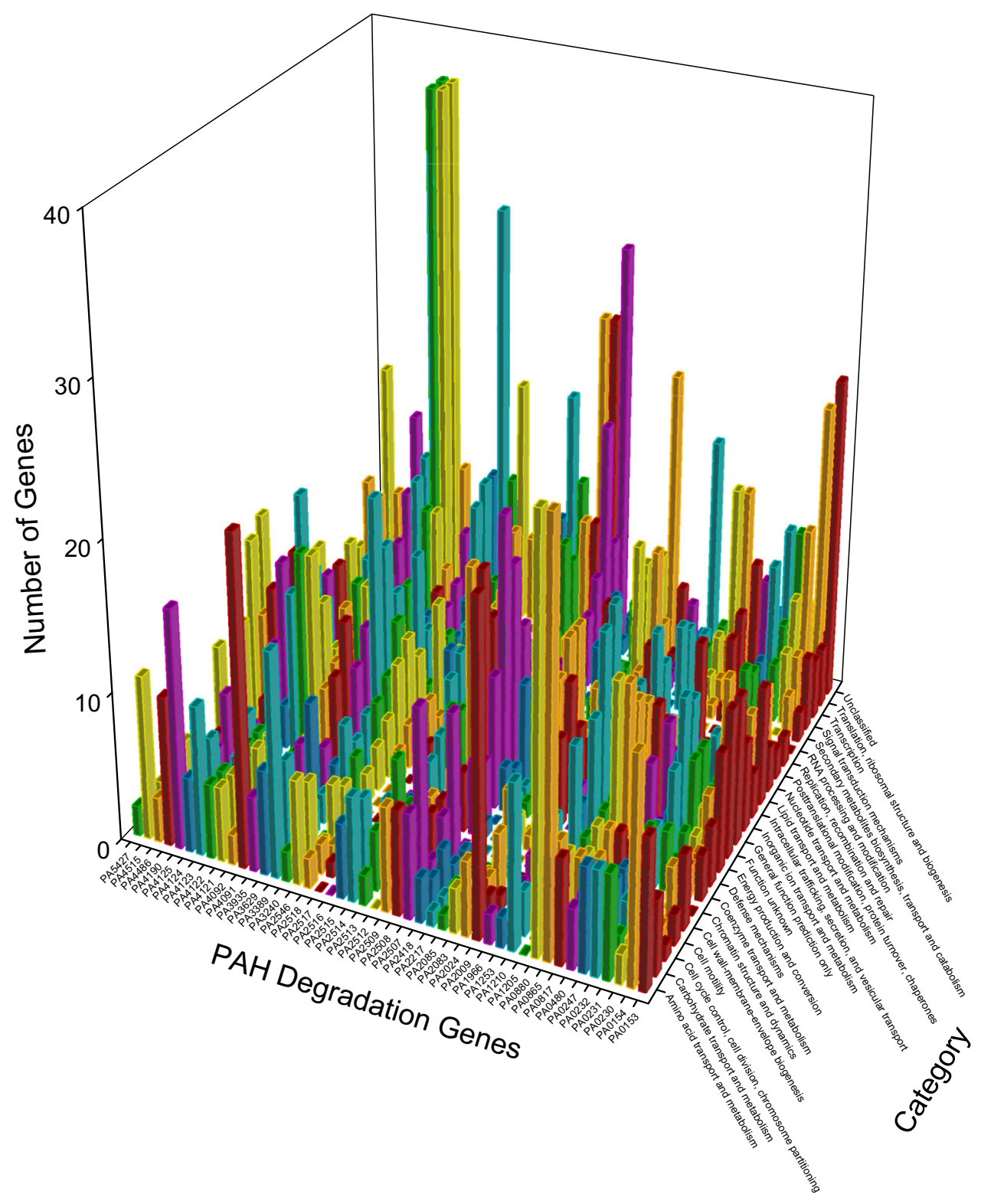

conditions appears more reasonable. Therefore, our analysis could render the knowledge on how PAH degradation genes work under different conditions. As mentioned in the Introduction, network analysis produces six novelties, which provide new information on PAH degradation.

\section{Comparing cluster numbers before and after stresses}

Network analysis groups the genes obtained from microarray into different clusters according to their correlation, and in this way the genes in a cluster have similar expression profiles, indicating that they encode interacting proteins, have a similar biological function, and belong to the same biological pathway (Braun 2014). Comparing cluster numbers before and after stresses or between different stresses, we can trace the co-actions of different genes. For example, network analysis discovers 19 clusters before the exposure (panel A), but 35 clusters after the exposure (panel B) in Figs. 1, and 38 clusters in Fig. 2, which is in good agreement with the experiment, where the exposure to sodium hypochlorite led to more changes in genes than the exposure to hydrogen peroxide (Small et al. 2007). As another example, network analysis uncovers the difference between top and bottom subpopulations of biofilms in Fig. 6 because there are more clusters in the upper panel than in the middle panel, which implies a higher activity in the top subpopulation of biofilms than in the bottom subpopulation. Similar observations can be found in other experimental conditions. Also, different strains can influence biofilm formation, and this feature can be remarkably presented in Fig. 8, where it is seen that the number of reorganized genes are bigger in panel 

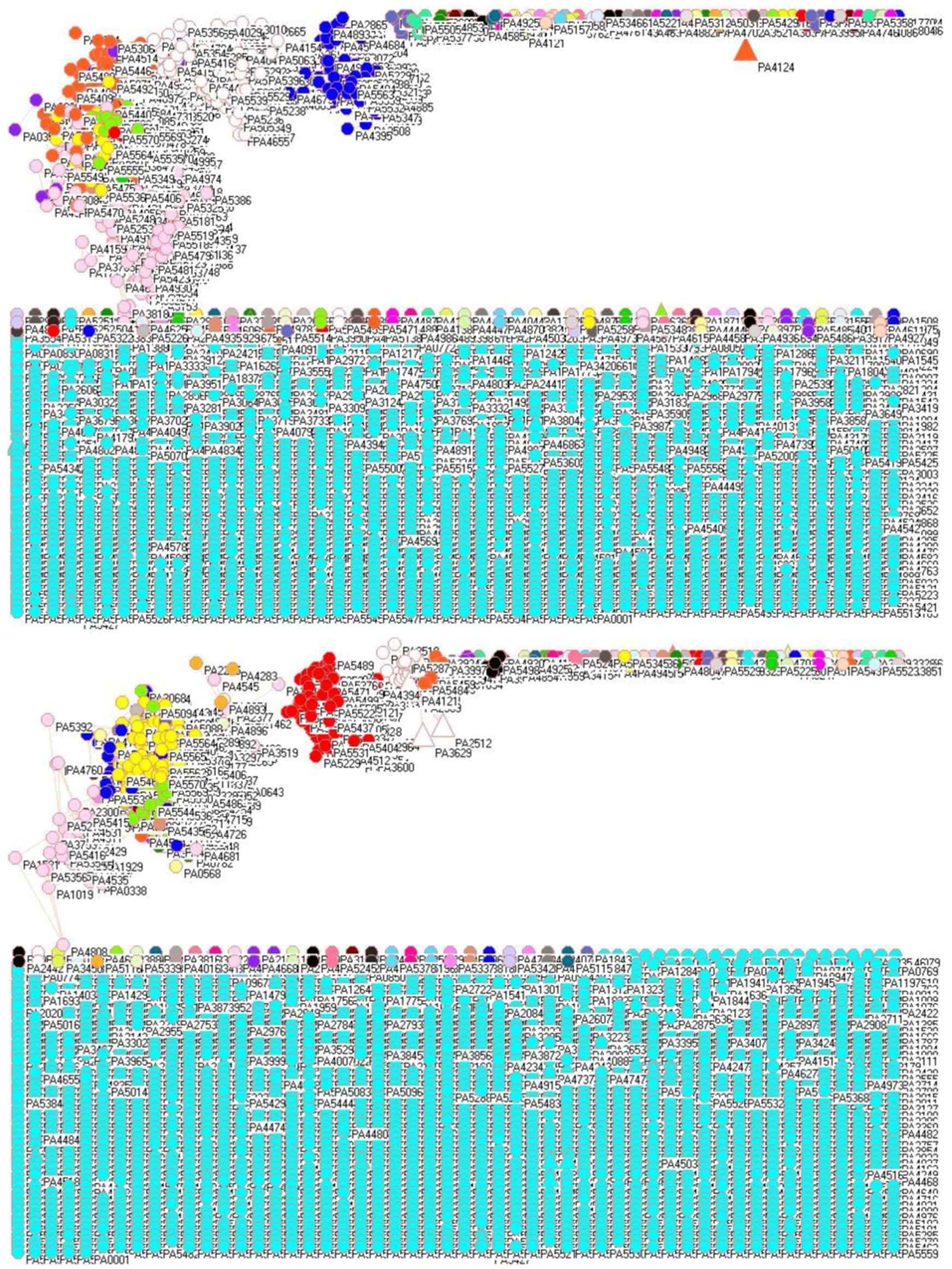
Fig. 3 Gene networks of $P$. aeruginosa PAO1 after oxygen exposure from high to low oxygen concentration (upper panel) and from low to high oxygen concentration (lower panel)

A than in panel B. Indeed, it was observed that biofilm formed by AES-1 is larger and thicker than usual (Manos et al. 2008).

\section{Comparing the membership in each cluster before and after stresses}

Network analysis provides insights on gene membership in each cluster and how it changes between control and treated groups, and between two subpopulations. For example, the lower panel in Fig. 6 displays such analysis, from which we can see how many genes changed their membership spatially in biofilms. Various reasons were given to explain why cells in biofilms spatially differ (Stewart and Franklin 2008), thus it is intriguing to find out whether PAH degradation genes follow such trend. Of 46 PAH degradation genes, 12 genes (PA0154, PA0817, PA0865, PA0880, PA1205, PA1210, PA1966, PA2024, PA2085, PA2217, PA2509, and PA3935) stay in their original clusters, whereas the other 34 genes changed their memberships. In addition, those $34 \mathrm{PAH}$ degradation genes are likely to be associated with high mRNA abundances including the genes PA4463, PA0059, PA2146, and PA3531. Taking Fig. 8 as another example, PA0634 and PA0635 in panel A are associated with a PAH degradation gene PA0865, and PA0639 is associated with two PAH degradation genes PA4123 and PA5427. This suggests the initiation of PAH degradation genes could go through the toxin-antitoxin principle (Dziewit et al. 2007) because the genes PA0632-PA0639 are identical to the tail proteins of bacteriophage N15 (Ravin et al. 2000), which acts along this line. A motility gene PA1094, which can change flagellar shape affecting motility, is associated with a PAH degradation gene PA4092. Also, an ABC transport system gene PA1247 is found to be associated with a PAH degradation gene PA4125. Four PAH degradation genes PA0154, PA0247, PA4121, and PA4122 have the same profiles in panel A, and they are associated with PA2386, a virulence factor pyoverdine gene that plays an important role in cystic fibrosis (Lamont et al. 2002).

\section{Defining which gene changed its membership together with PAH degradation genes}

On the one hand, many genes changed their membership together with PAH degradation genes when genes reorganized themselves under a certain condition. These associations are
A

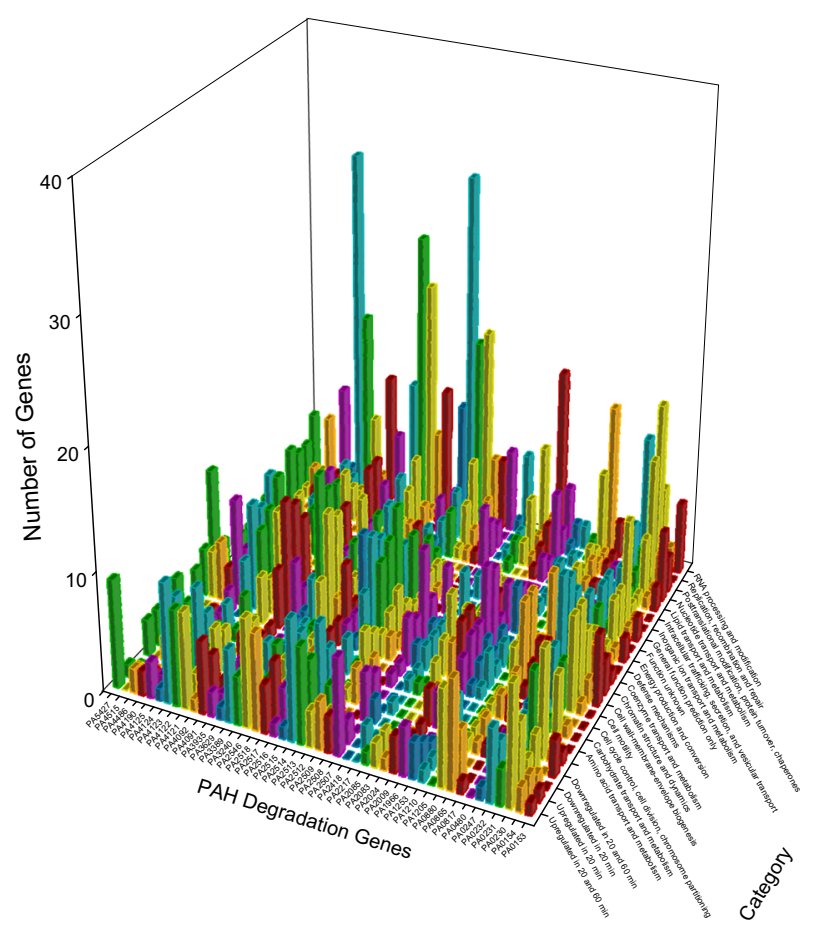

Fig. 4 Genes together with PAH degradation genes found in new clusters before and after exposure to ortho-phenylphenol in P. aeruginosa PAO1 for $20 \mathrm{~min}(\mathrm{~A})$ and for $60 \mathrm{~min}$ (B). In panel A, each pair of the following PAH degradation genes (PA0232 and PA4091, PA0247 and PA0817, PA0880 and PA2518, PA2507 and PA4121, PA2513 and PA4122,
B

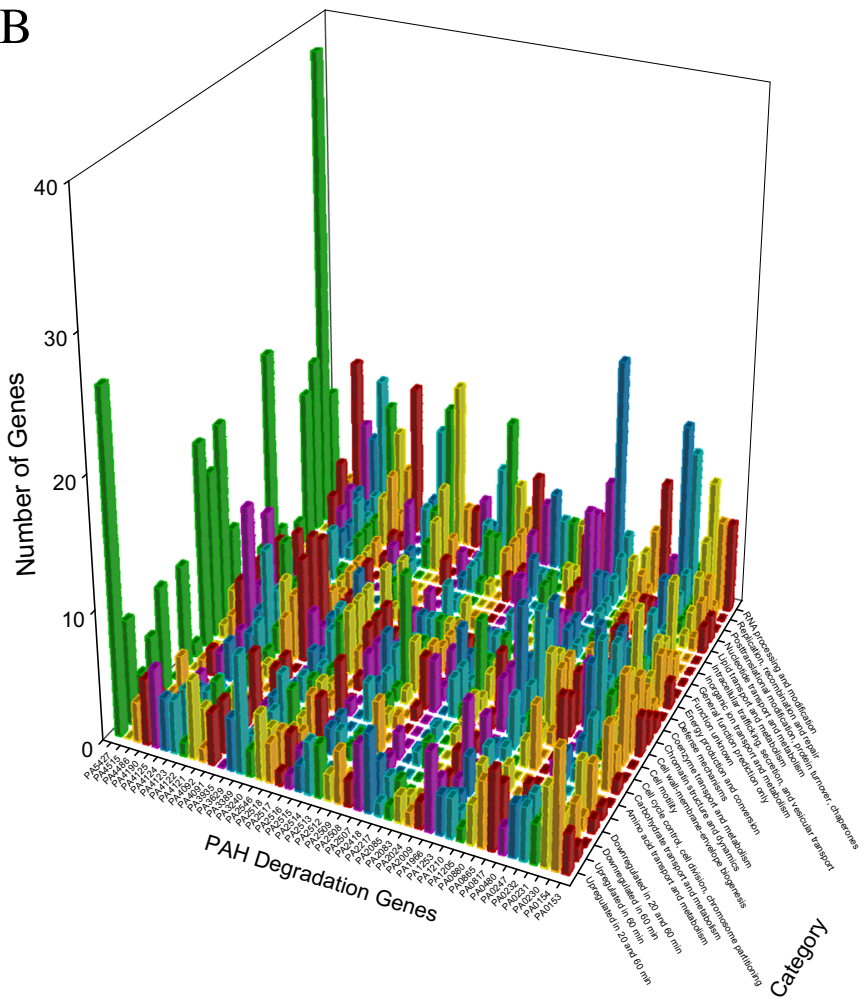

PA2514 and PA4123, PA3935 and PA4486) have the same profiles. In panel B, each pair of the following PAH degradation genes (PA0232 and PA4123, PA2217 and PA2516, PA2514 and PA4124, PA2518 and PA4486, PA2546 and PA4190) have the same profiles 
Fig. 5 Genes together with PAH degradation genes found in new clusters before and after expression of RpoN (PA4462) molecular roadblock in P. aeruginosa PAO1. PA0817 and PA2217 have the same profiles

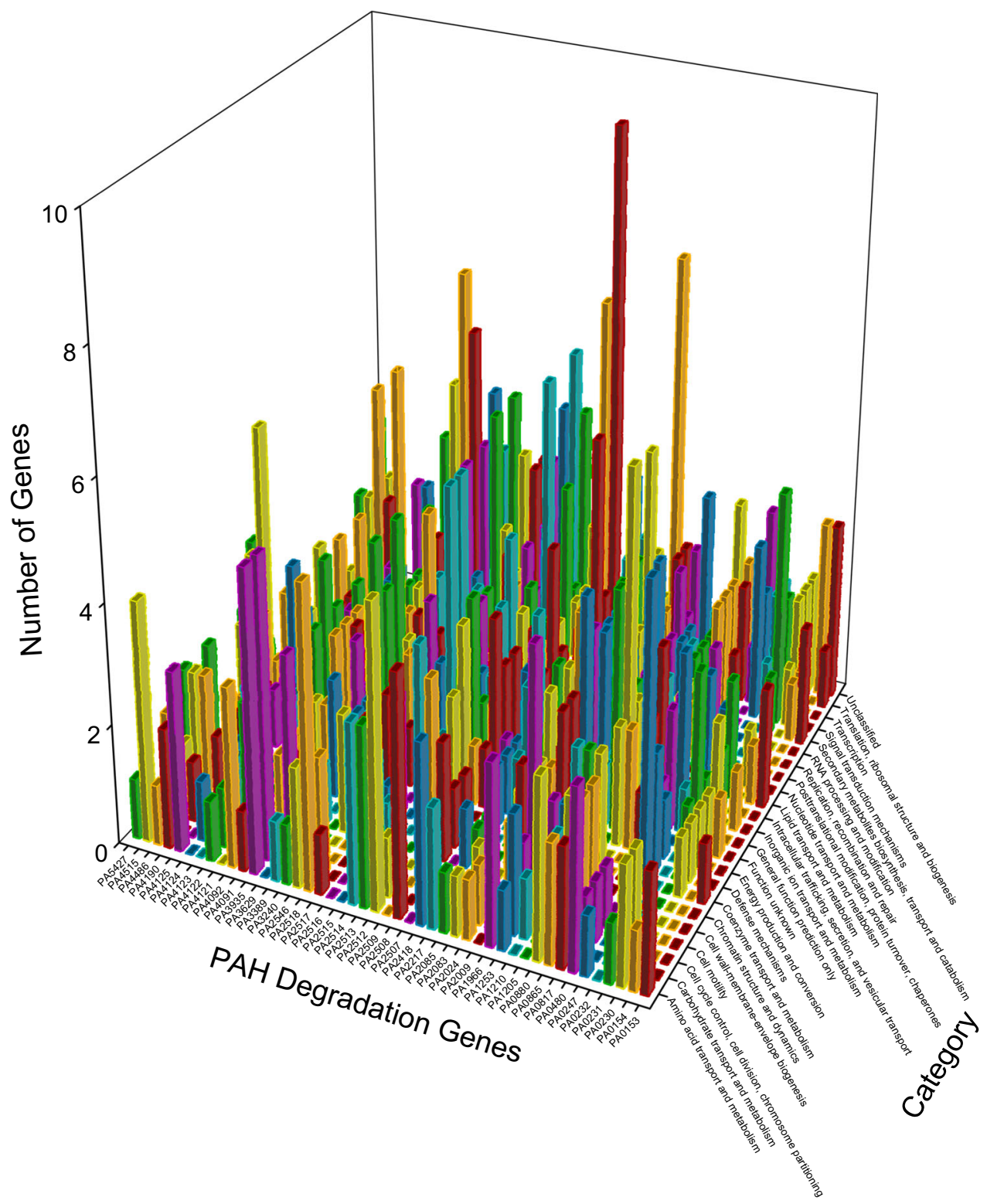

clearly demonstrated in panel C of Fig 1, and Figs. 2, 4, 5, 7 , and 8. For instance, PA0576 (RpoD) is a housekeeping gene because of its stability (Savli et al. 2003), and it changed its membership with a PAH degradation gene (PA1966) and 20 other genes to cluster purple from cluster olive green in Fig. 1. Of the 30 most downregulated genes in the exposure to hydrogen peroxide (Chang et al. 2005), two genes PA5479 and PA5446 appeared in two new clusters with PAH degradation genes PA2508 and PA4121, respectively, so network analysis does penetrate into different mechanisms. Of highly up/downregulated genes in response to oxidative stress, PA0182 encoding 3-ketoacyl-ACP reductase and PA2288 were associated with a PAH degradation gene PA0230, PA3008 encoding cell division inhibitor SulA was associated with the PAH degradation gene PA0231, PA5479 encoding glutamate/ aspartate:proton symporter was associated with the $\mathrm{PAH}$ degradation gene PA2508. Notably, PAH degradation gene PA4124 was associated with R2/F2 pyocin gene locus (PA0612-PA0648) because ten genes (PA0615, PA0616, PA0617, PA0620, PA0634-PA0638, and PA0641) from this locus together with PA4124 changed to cluster olive green from cluster cyan, implying that PA4124 works with R2/F2 pyocin gene locus. In biofilm, attention is paid to drug efflux pumps, of which PA4206 is associated with PAH degradation gene PA0154 (Fig. 7), therefore the initiation of PA0154 could

Fig. 6 Gene networks of the top $30 \mu \mathrm{m}$ (upper panel) and bottom $30 \mu \mathrm{m}$ (middle panel) of the $P$. aeruginosa PAO1 biofilms with PAH degradation genes (big symbols). Lower panel is gene compositions of the first five clusters (cluster 0 contains 1764 genes, cluster 1 contains 1730 genes, cluster 2 contains 1516 genes, cluster 3 contains 398 genes, cluster 4 contains 90 genes) in the bottom subpopulation of biofilms in terms of gene clusters in the top subpopulation of biofilms 


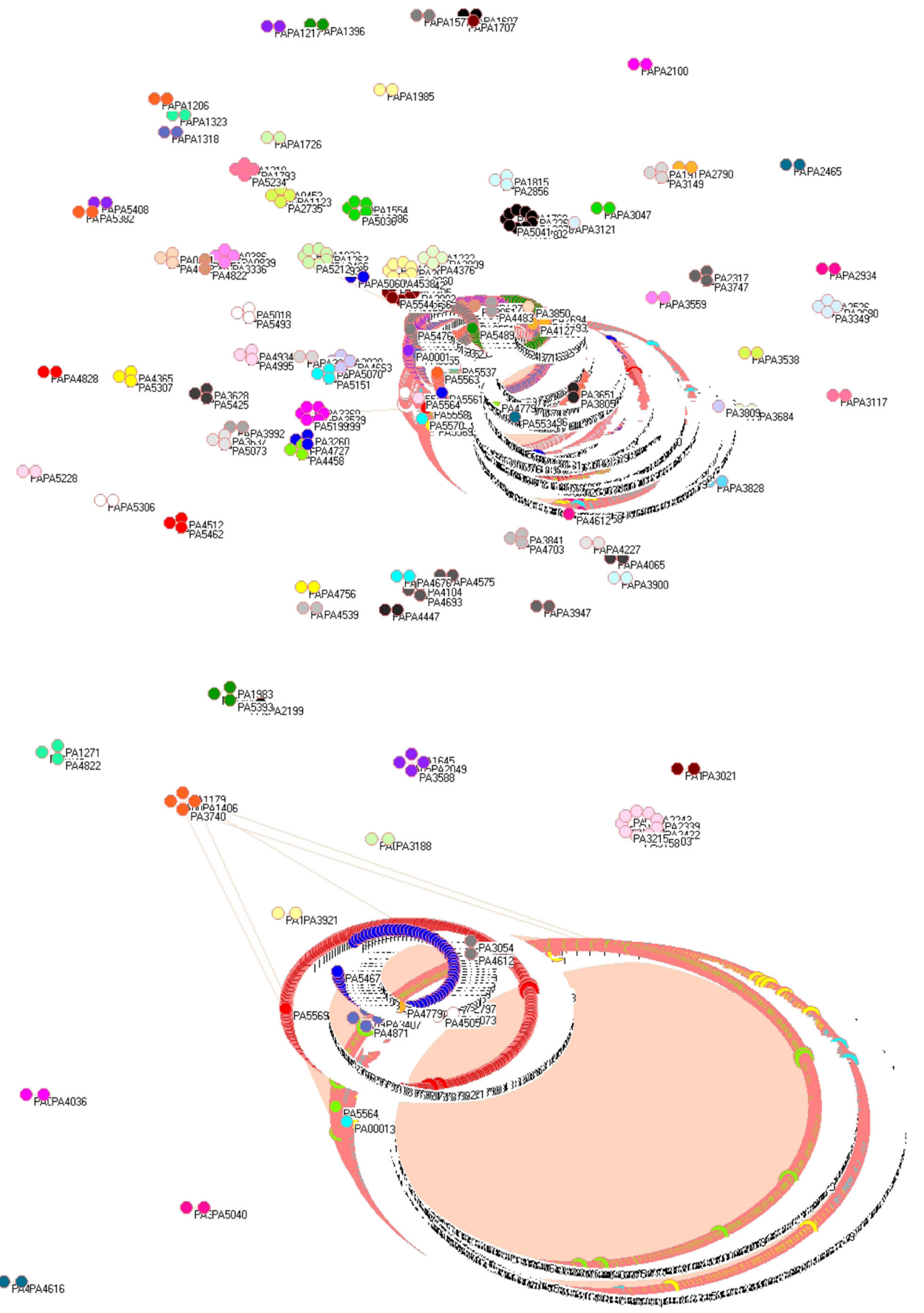



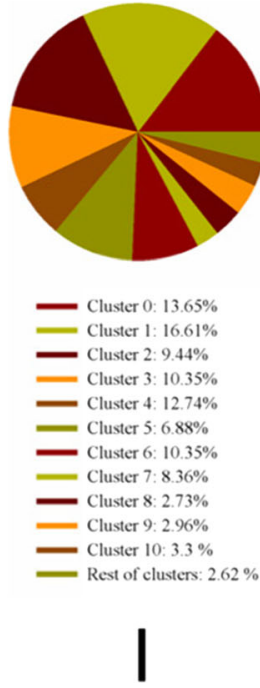

Fig. 6 (continued)
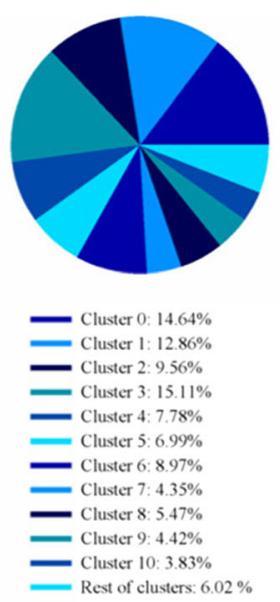

II
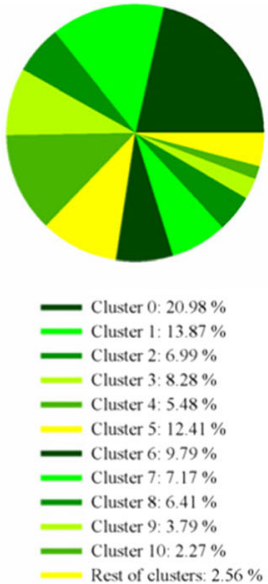

III
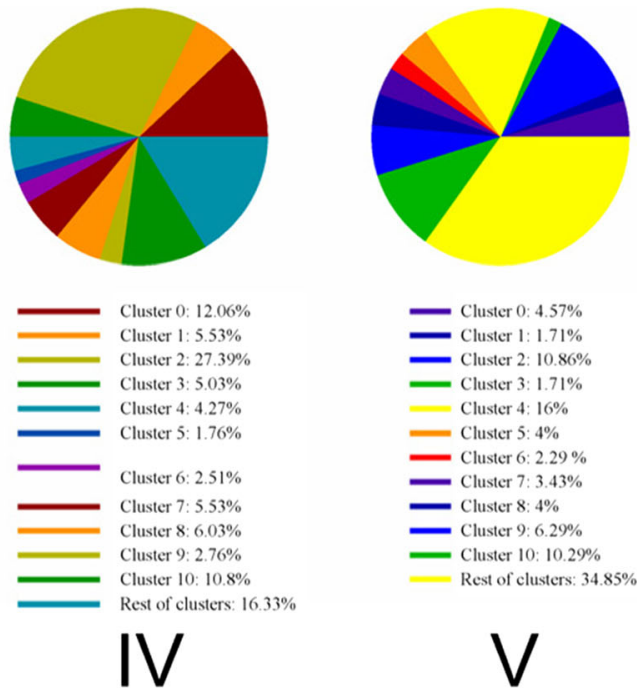

V relate to PA4206. In ciprofloxacin treatment (left-hand panel in Fig. 7), 18 PAH degradation genes (PA0231, PA0232, PA0480, PA0865,PA0880, PA1210, PA1966, PA2024, PA2507, PA2513, PA2514, PA2518, PA3240, PA3935, PA4091, PA4092, PA4122, and PA4125) are only associated with upregulated genes, whereas five PAH degradation genes (PA0247, PA1205, PA2516, PA2546, and PA5427) are only associated with downregulated genes. As ciprofloxacin stimulates the genes for SOS response, non-membrane-bound organelles, cytolysis and bacteriocin biosynthesis, so the first 18 PAH degradation genes would relate to these responses. In tobramycin treatment (right-hand panel in Fig. 7), $11 \mathrm{PAH}$ degradation genes (PA0230, PA0480, PA1253, PA2083, PA2217, PA4121, PA4122, PA4123, PA4125, PA4190, and PA 4515) are only associated with upregulated genes, whereas 11 PAH degradation genes (PA0153, PA2024, PA2085, PA2509, PA2514, PA2516, PA2546, PA3935, PA4092, PA4486, and PA5427) are only associated with downregulated genes. As tobramycin stimulates the genes for ribosome biosynthesis and RNA metabolism, and inhibits the genes for energy production, PAH degradation genes could be extrapolated along these directions. These findings once again demonstrate the importance of network analysis because the genes upregulated by ciprofloxacin and tobramycin did not overlap upregulated genes in biofilms (Stewart et al. 2015) but they were found to be associated with PAH degradation genes. In biofilm and quorum sensing studies, attention was given to the role of stationary-phase sigma factor RpoS (PA3622) (Schuster et al. 2004), which together with a PAH degradation gene PA2009 was found in a new cluster, suggesting their association. Also, a quorum sensing gene (PA1432, lasI) was found in a new cluster with PA0232, PA0880, and PA2517, which have the same profiles in both panels. In fact, a PAH degradation gene PA5427 was upregulated in several biofilm studies (Stewart et al. 2015). Those studies illustrated the function of some genes, and this study highlighted their association with PAH degradation genes. For instance, PA1555 encoding for a cytochrome c oxidase appears with a PAH degradation gene PA1966, and PA2386 encoding L-ornithine $\mathrm{N}^{5}$-oxygenase was associated with a PAH degradation gene PA4122.

On the other hand, some conditions did not lead many genes to regroup with PAH degradation genes, such as the exposure to RpoN roadblock (Fig. 5). This means that RpoN roadblock is very specific and does not interrupt many processes per se although sigma factor RpoN ( $\sigma 54)$ controls a regulon that is composed of around 20\% P. aeruginosa PAO1 genome (Damron et al. 2012), of which 59 genes from PA2134 to PA2192 are often studied. Intriguingly, if we look at genes PA2850, PA4613, PA4763, and PA5530 in panel C in Fig. 1, which were most highly regulated in the exposure to hydrogen peroxide (Chang et al. 2005), they do not appear in any new clusters with PAH degradation genes, this is the evidence that network analysis reveals different aspects of gene dynamics. Notably, PA0153, PA1210, PA1253, PA4125, and PA4190 in the left panel of Fig. 7, and PA0865, PA1205, PA1210, PA2518, PA3240, PA4091, and PA4123 in the right-hand panel are independent of the influence of upregulated genes in biofilms because none of the 340 upregulated genes in bioifilms appear in the first row for these PAH degradation genes. Furthermore, PA1201, PA4190, and PA5427 in the left-hand panel of Fig. 7, and PA0480 and PA1210 in the right-hand panel are independent of the influence of downregulated genes in biofilms because none of the 683 downregulated genes in bioifilms appear in the second row for these PAH degradation genes. In biofilms, the reduction of anabolic and catabolic activities is common, so the PAH degradation genes, which are independent of the influence of downregulated genes, should not be associated with catabolic pathways such as tricarboxylic acid cycle, NADH dehydrogenase, and cellular 


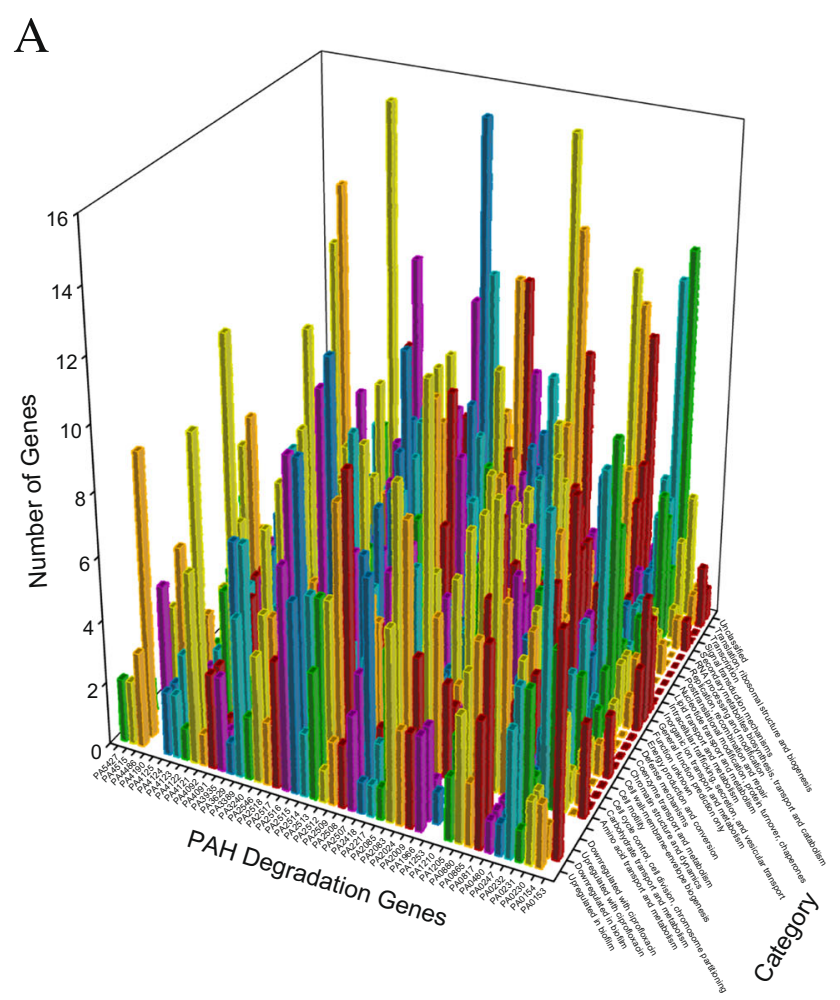

Fig. 7 Genes together with PAH degradation genes found in new clusters after $12 \mathrm{~h}$ treatments of the P. aeruginosa PAO1 biofilms with ciprofloxacin (left panel) and tobramycin (right panel). In left panel, each pair of the following PAH degradation genes (PA0232 and PA0880, PA0865 and PA2514, PA2024 and PA4486, PA2418 and

respiration. Because the location of PA2449 is close to the cluster of genes that are involved in glycine and serine metabolism (PA2442, PA2446, PA2493, and PA4315), however, these genes did not regroup together with $\mathrm{PAH}$ degradation genes (Fig. 9), so we can exclude that PAH degradation genes have any association with glycine and serine metabolism.

\section{Classifying membership-changed-genes}

Pseudomonas genes were classified into different categories in Pseudomonas Genome Database (Winsor et al. 2011, 2016), by which we can further analyze the membership-changed-genes to summarize their functions, as panel C in Fig. 1, and Figs. 2, 4, 5,7 , and 8 show such analysis. The exposure of hydrogen peroxide led $P$. aeruginosa PAO1 to have 223 genes significantly up/down-regulated, which were considered to relate to pyocin system genes and iron regulation (Chang et al. 2005). Taking panels A and B in Fig. 4 as an example, an interesting trend of gene reorganization can be observed along the time course. The genes with transport and metabolism functions are activated at the early time response to a stimulus, while many genes having functions of translation, ribosomal structure, and biogenesis are activated at the later time. Considering different stimuli or stresses, an integrated view can be found from

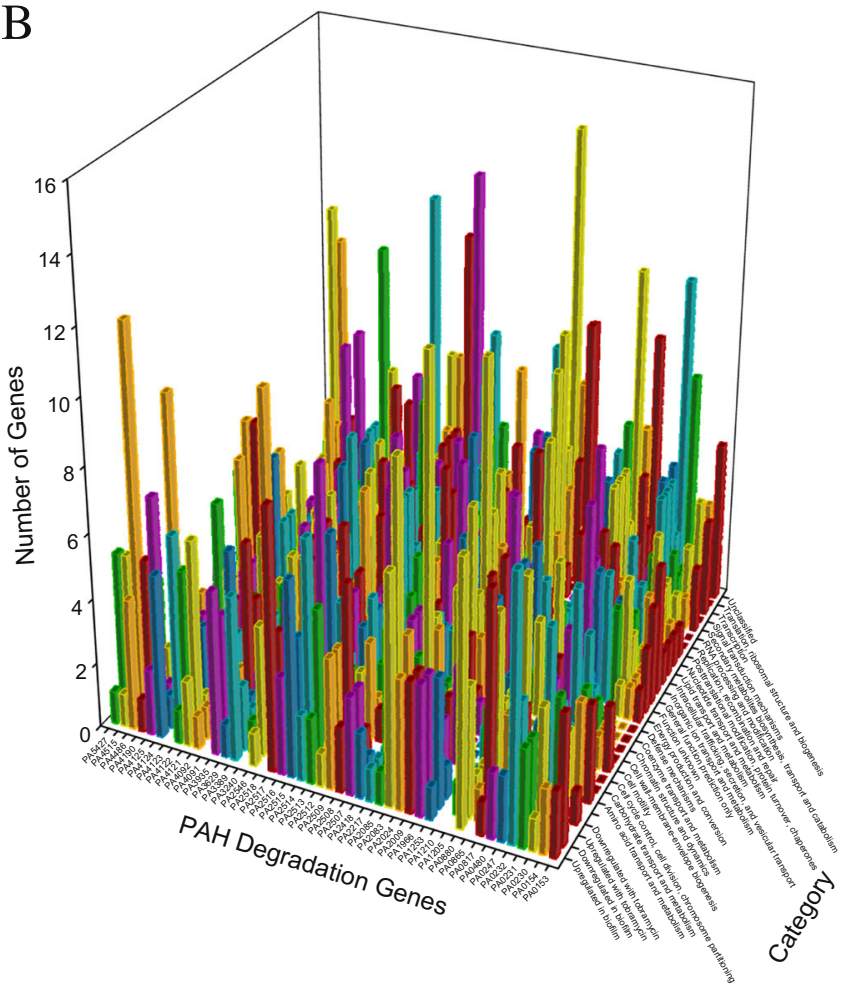

PA3629) have the same profile, and three genes PA2083, PA2515, and PA4121 have the same profile. In the right panel, each pair of the following PAH degradation genes (PA0247 and PA2418, PA0865 and PA1205, PA2085 and PA5427) have the same profiles, and three genes PA0232, PA0880, and PA2517 have the same profile

top to bottom in Fig. 10 where PAH degradation genes reveal 1) strong connections with the genes of amino acid transport and metabolism (the 3rd row), 2) more connections with the genes of inorganic ion transport and metabolism, energy production and conversion, lipid transport and metabolism, and transcription (the 4th to 7th rows), 3) moderate connections with the genes of posttranslational modification, protein turnover, chaperones, coenzyme transport and metabolism, signal transduction mechanisms, cell wall-membrane-envelope biogenesis, and carbohydrate transport and metabolism (the 8th to 12th rows), 4) fewer connections with the genes of replication, recombination and repair, nucleotide transport and metabolism, cell motility, secondary metabolites biosynthesis, transport and catabolism, and translation, ribosomal structure and biogenesis (the 13th to 17 th rows), and 5) fewer connections with the genes of defense mechanisms, intracellular trafficking, secretion, and vesicular transport, cell cycle control, cell division, chromosome partitioning, chromatin structure and dynamics, and RNA processing and modification (the 18th to 22 nd rows).

\section{Postulating unknown gene's function}

Actually, many P. aeruginosa PAO1 genes have yet to define their functions. The detailed analysis of the associations of genes 


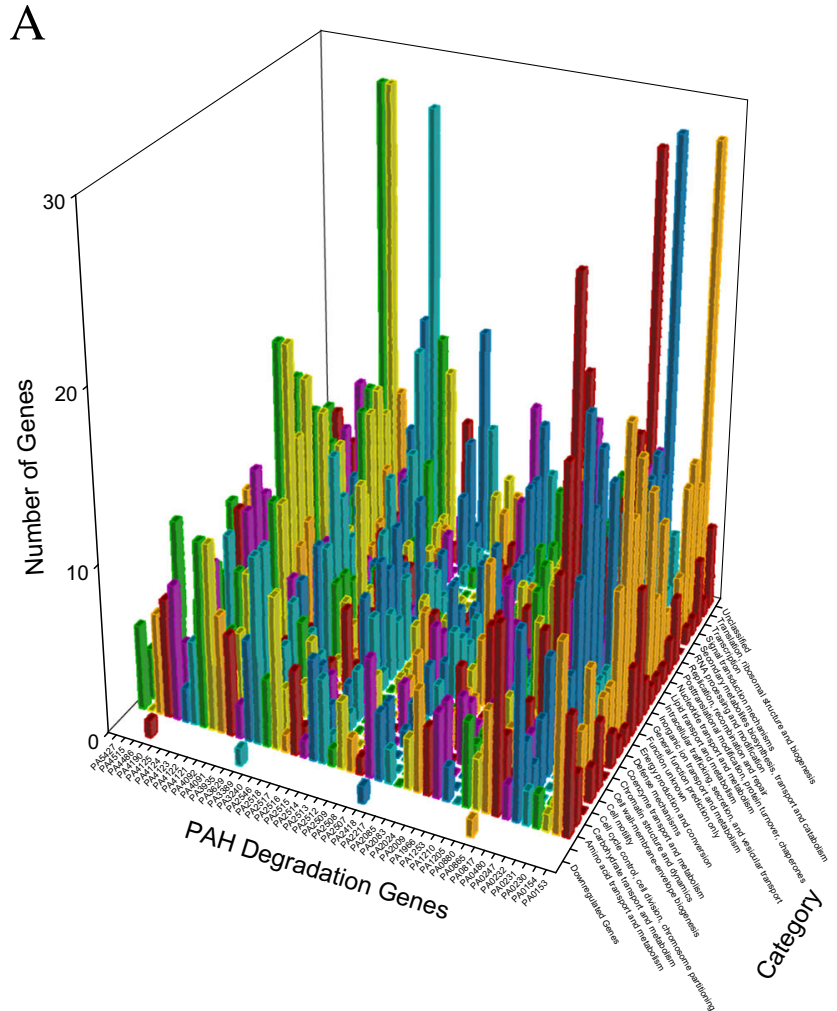

Fig. 8 Genes together with PAH degradation genes found in new clusters of $P$. aeruginosa AES-1 (A) and AES-2 (B) in biofilm. In panel A, each pair of the following PAH degradation genes (PA2009 and PA3240, PA2514 and PA2517, PA2515 and PA3629, PA4123 and PA5427) have the same profiles, and the genes PA0154, PA0247, PA4121, and PA4122

when changing their membership can get an idea on unknown gene's functions. Network analysis uncovered some unknown function genes having association with PAH degradation genes. For example, the unknown function genes PA1746, PA2782 (Stewart et al. 2015), and PA3572 had association with PAH degradation genes PA3629, PA0817, and PA2516, respectively. An unknown function gene PA1673 and a phenazine biosynthesis protein PA4211 were associated with a PAH degradation gene PA3389. An unknown function gene PA0713 and PA4765 encoding outer membrane lipoprotein OmlA precursor were associated with a PAH degradation gene PA4486.

Furthermore, the gene PA0247 in panels A and B in Fig. 1 encodes a 4-hydroxybenzoate 3-monooxygenase, and belonged to the light green cluster before the exposure (panel A) but changed to the black cluster after the exposure (panel B). Interestingly, it did not act alone, but with eight other genes (PA0470, PA0863, PA1417, PA2494, PA2635, PA3502, PA4649, and PA4660), i.e., these eight genes changed their membership from the light green cluster to the black one (Supplementary materials). Of these eight genes, PA0470 encodes ferrichrome receptor FiuA, PA0863 encodes an oxidoreductase, PA2494 encodes resistance-nodulation-cell division (RND) multidrug efflux transporter MexF, and PA4660 encodes a deoxyribodipyrimidine photolyase, while the functions of

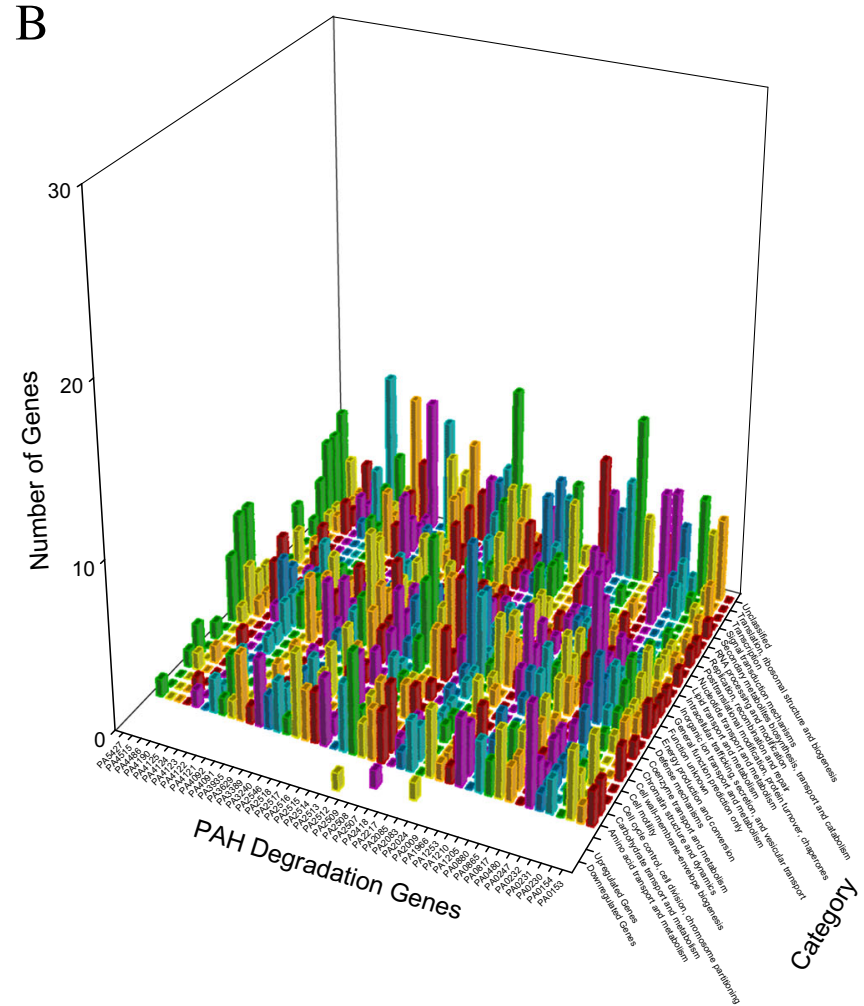

have the same profiles. In panel $\mathrm{B}$, each pair of the following PAH degradation genes (PA0153 and PA0865, PA0880 and PA2516, PA2508 and PA2509, PA3935 and PA4092, PA4091 and PA4122) have the same profiles

PA1417, PA2635, PA3502, and PA4649 are unknown. Therefore, we can not only postulate that PA0247 initiation needs the transporter, receptor, oxidoreductase and photolyase, but also have the clue for the function of undefined genes. Taking a hypothetical protein PA4486 as another example, it located on the far right-hand side of Fig. 10, branched together with PA2024 (ring-cleaving dioxygenase) and then branched with PA2513 (ant $B$, anthranilate dioxygenase small subunit), suggesting that these three genes work together. Moreover, PA4486 was found to be involved in the degradation of aromatic compounds for cleavage of a ring in reaction from 4-carboxymuconolactone to 2-oxo2,3dihydrofuran-5-acetate and in the degradation of benzoate for cleavage of a ring in reaction between $\gamma$-carboxymuconolactone and 3-oxoadipateenol-latone. Therefore, these three genes, PA2024, PA2513, and PA4486, are more likely to be initiated by the genes in blue, indigo, and violet colors in the right-upper corner of Fig. 10 for those two reactions. Similar deduction and reasoning can be applied to all the PAH degradation genes in question.

\section{Proposing a new mechanism on genes of interests}

The comparison between control and experimental groups in each experiment does generally provide a way to understand 

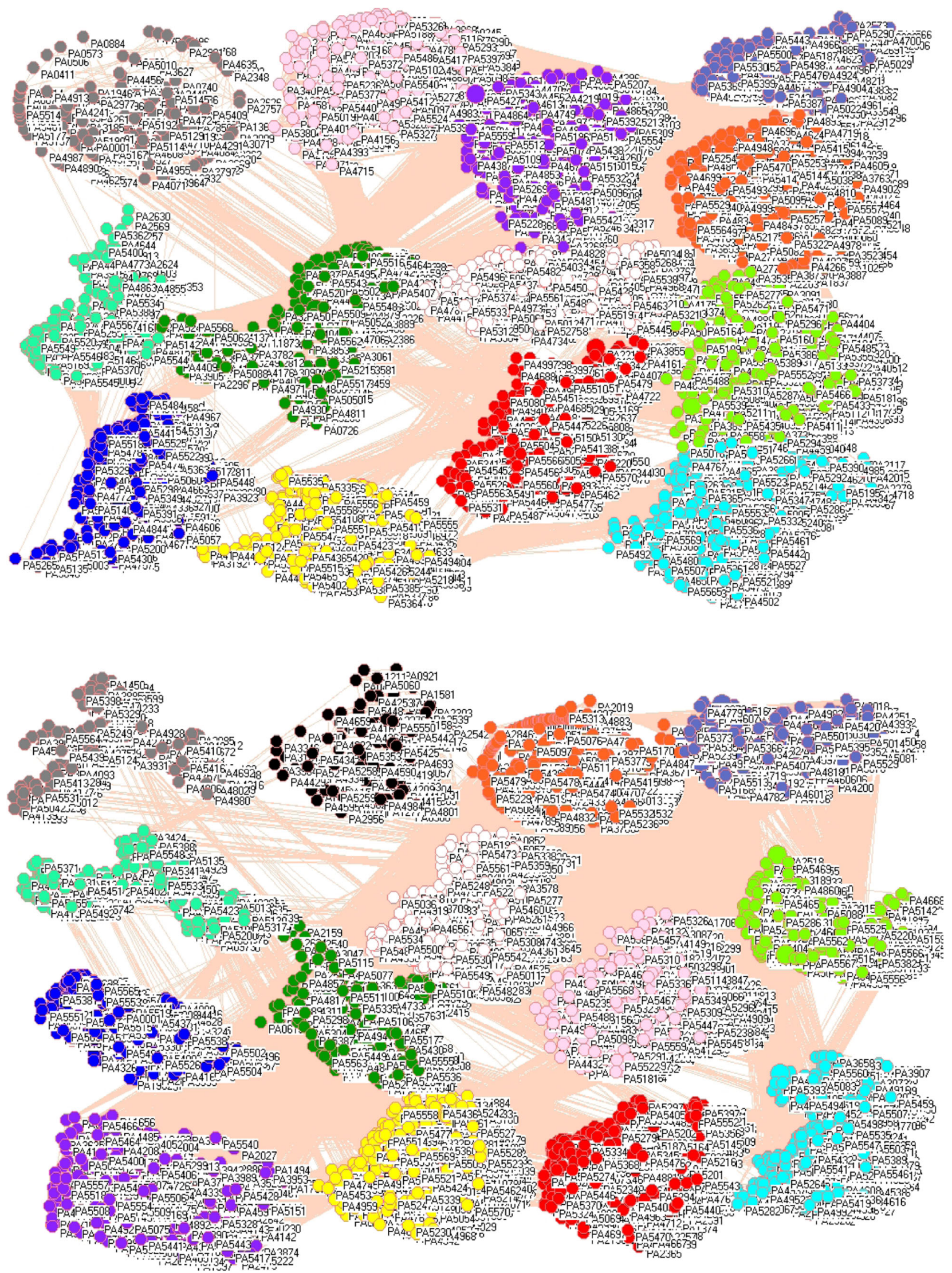

Fig. 9 Gene networks of wild-type P. aeruginosa PAO1 (upper panel) and PA2449-null mutant P. aeruginosa PW5126 (lower panel) 


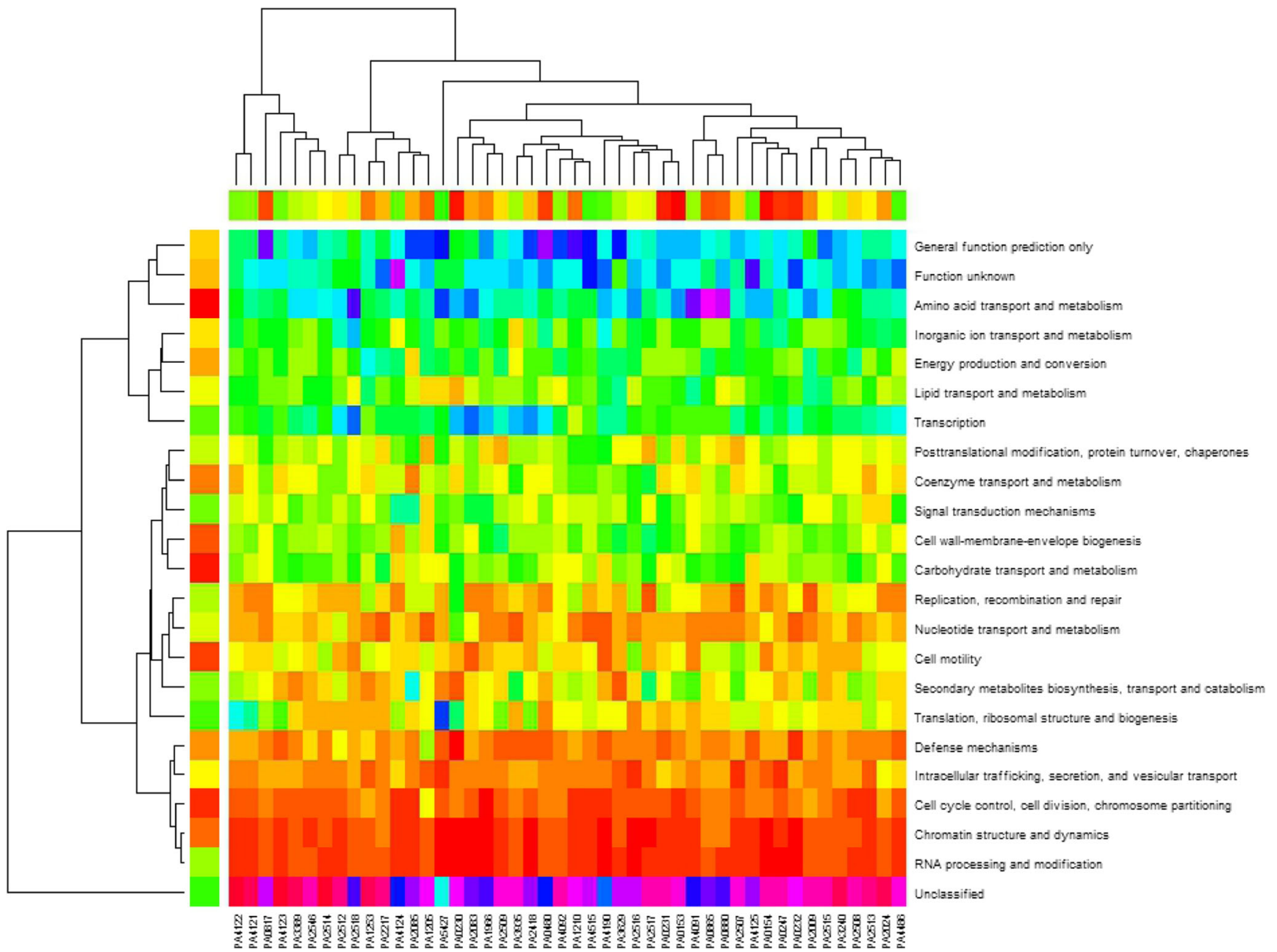

Fig. 10 Heatmap and cluster analysis of PAH degradation genes and their association with gene category in response to nine different stresses and conditions. Different colors in heatmap represent different numbers of genes in terms of gene category, and trees of dendrogram classify PAH degradation genes and gene category

encodes quercetin 2,3-dioxygenase, PA2009 encodes homogentisate 1,2-dioxygenase, PA2513 encodes anthranilate dioxygenase small subunit, PA4091 encodes 4hydroxyphenylacetate 3-monooxygenase large subunit, and PA4125 encodes 5-carboxymethyl-2-hydroxymuconate isomerase. So the close relationship between PAH degradation genes can be established through this metabolic pathway.

In this study, we analyzed just the PAH degradation genes although alkane degradation genes were sometimes analyzed together with PAH degradation genes (Liu et al. 2012; Gai et al. 2012) because PAH is a polycyclic aromatic hydrocarbon while hazardous alkanes are generally linear structured, i.e., methane, ethane, propane, and butane. Naturally they have different metabolic pathways. For PAHs, the opening of the aromatic ring is important, for example, PA0817 encodes an aromatic ring-cleaving dioxygenase. When considering the influence of different oxygen concentrations in Fig. 3, it is interesting to find that 17 PAH degradation genes (PA0230, PA0247, PA0480, PA0817, PA1205, PA1210, PA2024, PA2418, 
PA2509, PA3389, PA3935, PA4092, PA4123, PA4125, PA4486, PA4515, and PA5427) were not associated with any other genes when oxygen concentration went from high to low (upper panel), while six PAH degradation genes (PA4091, PA4123, PA4125, PA4486, PA4515, and PA5427) were not associated with any other genes when oxygen concentration went from low to high (lower panel). In fact, the isolated genes included an important well-known oxygen responding gene PA5171, whereas the other three well-known oxygen responding genes PA5170, PA5172, and PA5173 were located in the lime green cluster (lower panel), and they were associated with seven PAH degradation genes PA0153, PA0480, PA0817, PA2009, PA2517, PA2518, and PA3240. Of the 85 genes identified as essential oxygen-availability response genes (He et al. 2014), PA0230 and PA4515 were PAH degradation genes.

In Fig. 8, two quorum-sensing genes PA3049 and PA3922 are associated with two PAH degradation genes PA0865 and PA2418, respectively. Again, eight genes (PA0958, PA1432, PA1713, PA1723, PA1777, PA1947, PA4922, and PA5253) were focused in AES-2, however, they are not associated with any PAH degradation genes to change their membership (Supplementary materials). AES-2 biofilms had upregulated genes in the type III secretion system, but PA1723, PA1713, and PA1705 are not associated with any PAH degradation genes (panel B), so PAH degradation genes would not interact with the type III secretion system.

\section{Conclusions}

In this study, network analysis was used to analyze how 46 PAH degradation genes reorganized among 5549 genes in P. aeruginosa PAO1 under nine different conditions and stresses. The results demonstrate the heterogeneity of gene networks and regrouping of genes under different conditions. The hierarchical structures between PAH degradation genes and P. aeruginosa PAO1 genes with different functions were stratified by dendrogram trees. Six aspects of novelties produced through network analysis are discussed in detail. To our knowledge, this is the first study to use network analysis to investigate synthetically how PAH degradation genes reorganized under different conditions. Such studies can shed lights on understanding gene interactions and reorganizations under various conditions and environmental stresses, which pave the way to conduct similar studies on other genes.

\section{Compliance with ethical standards}

Funding This study was partly supported by research grants from National Natural Science Foundation of China (31460296 and 31560315), and Special Funds for Building of Guangxi Talent Highland.
Conflict of interest Authors declare that they have no conflict of interest.

Ethical approval This article does not contain any studies with human participants or animals performed by any of the authors.

Open Access This article is distributed under the terms of the Creative Commons Attribution 4.0 International License (http:// creativecommons.org/licenses/by/4.0/), which permits unrestricted use, distribution, and reproduction in any medium, provided you give appropriate credit to the original author(s) and the source, provide a link to the Creative Commons license, and indicate if changes were made.

\section{References}

Balashova NV, Stolz A, Knackmuss HJ, Kosheleva IA, Naumov AV, Boronin AM (2001) Purification and characterization of a salicylate hydroxylase involved in 1-hydroxy-2-naphthoic acid hydroxylation from the naphthalene and phenanthrene-degrading bacterial strain Pseudomonas putida BS202-P1. Biodegradation 12:179-188

Barrett T, Wilhite SE, Ledoux P, Evangelista C, Kim IF, Tomashevsky M, Marshall KA, Phillippy KH, Sherman PM, Holko M, Yefanov A, Lee H, Zhang N, Robertson CL, Serova N, Davis S, Soboleva A (2013) NCBI GEO: archive for functional genomics data sets-update. Nucleic Acids Res 41:D991-D995

Basta T, Buerger S, Stolz A (2005) Structural and replicative diversity of large plasmids from sphingomonads that degrade polycyclic aromatic compounds and xenobiotics. Microbiology 151:2025-2037

Bogan BW, Lamar RT (1996) Polycyclic aromatic hydrocarbons degrading capability of Phanerochaete laevis HHB-1625 and its extracellular ligninolytic enzymes. Appl Environ Microbiol 62: $1597-1603$

Bogan BW, Schoenike B, Lamar RT, Cullen D (1996) Expression of lip genes during growth in soil and oxidation of anthracene by Phanerochaete chrysosporium. Appl Environ Microbiol 62:36973703

Bosch R, Garcia-Valdes E, Moore ERB (2000) Complete nucleotide sequence and evolutionary significance of a chromosomally encoded naphthalene-degradation lower pathway from Pseudomonas stutzeri AN10. Gene 245:67-74

Braun R (2014) Systems analysis of high-throughput data. Adv Exp Med Biol 844:153-187

Cerniglia CE (1997) Fungal metabolism of polycyclic aromatic hydrocarbons: past, present and future applications in bioremediation. $\mathrm{J}$ Ind Microbiol Biotechnol 19:324-333

Chang W, Small DA, Toghrol F, Bentley WE (2005) Microarray analysis of Pseudomonas aeruginosa reveals induction of pyocin genes in response to hydrogen peroxide. BMC Genomics 6:115

Damron FH, Owings JP, Okkotsu Y, Varga JJ, Schurr JR, Goldberg JB, Schurr MJ, Yu HD (2012) Analysis of the Pseudomonas aeruginosa regulon controlled by the sensor kinase $\mathrm{KinB}$ and sigma factor RpoN. J Bacteriol 194:1317-1330

de Nooy W, Mrvar A, Batagelj V (2011) Exploratory social network analysis with Pajek: revised and expanded second edition, structural analysis in the social sciences. Cambridge University Press, Cambridge

Dziewit L, Jazurek M, Drewniak L, Baj J, Bartosik D (2007) The SXT conjugative element and linear prophage N15 encode toxin-antitoxin-stabilizing systems homologous to the tad-ata module of the Paracoccus aminophilus plasmid pAMI2. J Bacteriol 189:19831997 
Edgar R, Domrachev M, Lash AE (2002) Gene expression omnibus: $\mathrm{NCBI}$ gene expression and hybridization array data repository. Nucleic Acids Res 30:207-210

Ferreira LE, Muniz BV, Bittar TO, Berto LA, Figueroba SR, Groppo FC, Pereira AC (2014) Effect of particles of ashes produced from sugarcane burning on the respiratory system of rats. Environ Res 135: 304-310

Floehr T, Xiao H, Scholz-Starke B, Wu L, Hou J, Yin D, Zhang X, Ji R, Yuan X, Ottermanns R, Roß-Nickoll M, Schäffer A, Hollert H (2013) Solution by dilution?-a review on the pollution status of the Yangtze River. Environ Sci Pollut Res Int 20:6934-6971

Frazee RW, Livingston DM, LaPorte DC, Lipscomb JD (1993) Cloning, sequencing, and expression of the Pseudomonas putida protocatechuate 3,4-dioxygenase genes. J Bacteriol 175:6194-6202

Gai Z, Zhang Z, Wang X, Tao F, Tang H, Xu P (2012) Genome sequence of Pseudomonas aeruginosa DQ8, an efficient degrader of $\mathrm{n}$ alkanes and polycyclic aromatic hydrocarbons. J Bacteriol 194: 6304-6305

Ghiorse WC, Herrick JB, Sandoli RL, Madsen EL (1995) Natural selection of PAH-degrading bacterial guilds at coal-tar disposal sites. Environ Health Perspect 103(Suppl 5):107-111

Gordon L, Dobson AD (2001) Fluoranthene degradation in Pseudomonas alcaligenes PA-10. Biodegradation 12:393-400

Goyal AK, Zylstra GJ (1997) Genetics of naphthalene and phenanthrene degradation by Comamonas testosteroni. J Ind Microbiol Biotechnol 19:401-440

Guo S, Hu M, Zamora ML, Peng J, Shang D, Zheng J, Du Z, Wu Z, Shao M, Zeng L, Molina MJ, Zhang R (2014) Elucidating severe urban haze formation in China. Proc Natl Acad Sci U S A 111:1737317378

Habe H, Miyakoshi M, Chung JS, Kasuga K, Yoshida T, Nojiri H, Omori $\mathrm{T}$ (2003) Phthalate catabolic gene cluster is linked to the angular dioxygenase gene in Terrabacter sp. strain DBF63. Appl Microbiol Biotechnol 61:44-54

He FQ, Wang W, Zheng P, Sudhakar P, Sun J, Zeng AP (2014) Essential $\mathrm{O}_{2}$-responsive genes of Pseudomonas aeruginosa and their network revealed by integrating dynamic data from inverted conditions. Integr Biol (Camb) 6:215-223

Iwabuchi T, Harayama S (1998) Biochemical and molecular characterization of 1-hydroxy-2-naphthoate dioxygenase from Nocardioides sp. KP7. J Biol Chem 273:8332-8336

Kamerbeek NM, Moonen MJ, Van Der Ven JG, Van Berkel WJM, Fraaije W, Janssen DB (2001) 4-Hydroxyacetophenone monooxygenase from Pseudomonas fluorescens ACB. A novel flavoprotein catalyzing Baeyer-Villiger oxidation of aromatic compounds. Eur J Biochem 268:2547-2557

Kanaly RA, Harayama S (2000) Biodegradation of high-molecularweight polycyclic aromatic hydrocarbons by bacteria. J Bacteriol 182:2059-2067

Kanehisa M, Goto S (2000) KEGG: kyoto encyclopedia of genes and genomes. Nucleic Acids Res 28:27-30

Kanehisa M, Goto S, Sato Y, Kawashima M, Furumichi M, Tanabe M (2014) Data, information, knowledge and principle: back to metabolism in KEGG. Nucleic Acids Res 42:D199-D205

Kanehisa M, Sato Y, Kawashima M, Furumichi M, Tanabe M (2016) KEGG as a reference resource for gene and protein annotation. Nucleic Acids Res 44:D457-D462

Kim SJ, Kweon O, Jones RC, Freeman JP, Edmondson RD, Cerniglia CE (2007) Complete and integrated pyrene degradation pathway in Mycobacterium vanbaalenii PYR-1 based on systems biology. J Bacteriol 189:464-472

Kirk TK, Farrell RL (1987) Enzymatic "combustion": the microbial degradation of lignin. Annu Rev Microbiol 41:465-505

Kiyohara H, Nagao K, Kouno K, Yano K (1982) Phenanthrene-degrading phenotype of Alcaligenes faecalis AFK2. Appl Environ Microbiol $43: 458-461$
Kulakov LA, Allen CCR, Lipscomb DA, Larkin MJ (2000) Cloning and characterization of a novel cis-naphthalene dihydrodiol dehydrogenase gene (narB) from Rhodococcus $s p$. NCIMB 12038. FEMS Microbiol Lett 182:327-331

Kulakov LA, Chen SC, Allen CCR, Larkin MJ (2005) Web-type evolution of Rhodococcus gene clusters associated with utilization of naphthalene. Appl Environ Microbiol 71:1754-1764

Kweon O, Kim SJ, Jones RC, Freeman JP, Adjei MD, Edmondson RD, Cerniglia CE (2007) A polyomic approach to elucidate the fluoranthene-degradative pathway in Mycobacterium vanbaalenii PYR-1. J Bacteriol 189:4635-4647

Kweon O, Kim SJ, Kim DW, Kim JM, Kim HL, Ahn Y, Sutherland JB, Cerniglia CE (2014) Pleiotropic and epistatic behavior of a ringhydroxylating oxygenase system in the polycyclic aromatic hydrocarbon metabolic network from Mycobacterium vanbaalenii PYR1. J Bacteriol 196:3503-3515

Lamont IL, Beare PA, Ochsner U, Vasil AI, Vasil ML (2002) Siderophore-mediated signaling regulates virulence factor production in Pseudomonas aeruginosa. Proc Natl Acad Sci U S A 99: 7072-7077

Laurie AD, Lloyd-Jones G (1999) Conserved and hybrid meta-cleavage operons from PAH-degrading Burkholderia RP007. Biochem Biophys Res Commun 262:308-314

Lee J, Zhang L (2015) The hierarchy quorum sensing network in Pseudomonas aeruginosa. Protein Cell 6:26-41

Li W, Shi J, Wang X, Han Y, Tong W, Ma L, Liu B, Cai B (2004) Complete nucleotide sequence and organization of thenaphthalene catabolic plasmid pND6-1 from Pseudomonas $s p$. strain ND6. Gene 336:231-240

Liu H, Liang R, Tao F, Ma C, Liu Y, Liu X, Liu J (2012) Genome sequence of Pseudomonas aeruginosa strain SJTD-1, a bacterium capable of degrading long-chain alkanes and crude oil. J Bacteriol 194:4783-4784

Lundgren BR, Thornton W, Dornan MH, Villegas-Peñaranda LR, Boddy CN, Nomura CT (2013) Gene PA2449 is essential for glycine metabolism and pyocyanin biosynthesis in Pseudomonas aeruginosa PAO1. J Bacteriol 195:2087-2100

Manos J, Arthur J, Rose B, Tingpej P, Fung C, Curtis M, Webb JS, Hu H, Kjelleberg S, Gorrell MD, Bye P, Harbour C (2008) Transcriptome analyses and biofilm-forming characteristics of a clonal Pseudomonas aeruginosa from the cystic fibrosis lung. J Med Microbiol 57:1454-1465

Manos J, Arthur J, Rose B, Bell S, Tingpej P, Hu H, Webb J, Kjelleberg S, Gorrell MD, Bye P, Harbour C (2009) Gene expression characteristics of a cystic fibrosis epidemic strain of Pseudomonas aeruginosa during biofilm and planktonic growth. FEMS Microbiol Lett 292: $107-114$

Meckenstock RU, Safinowski M, Griebler C (2004) Anaerobic degradation of polycyclic aromatic hydrocarbons. FEMS Microbiol Ecol 49: $27-36$

Menn FM, Applegate BM, Sayler GS (1993) NAH plasmid-mediated catabolism of anthracene and phenanthrenen to naphthoic acids. Appl Environ Microbiol 59:1938-1942

Miller RA, Britigan BE (1997) Role of oxidants in microbial pathophysiology. Clin Microbiol Rev 10:1-18

Mueller JG, Chapman PJ, Blattmann BO, Pritchard PH (1990) Isolation and characterization of a fluoranthene-utilizing strain of Pseudomonas paucimobilis. Appl Environ Microbiol 56:10791086

Nam IH, Chang YS, Hong HB, Lee YE (2003) A novel catabolic activity of Pseudomonas veronii in biotransformation of pentachlorophenol. Appl Microbiol Biotechnol 62:284-290

Nde CW, Jang HJ, Toghrol F, Bentley WE (2008) Toxicogenomic response of Pseudomonas aeruginosa to ortho-phenylphenol. BMC Genomics 9:473 
Nojiri H, Maeda K, Sekiguchi H, Urata M, Shintani M, Yoshida T, Habe H, Omori T (2002) Organization and transcriptional characterization of catechol degradation genes involved in carbazole degradation by Pseudomonas resinovorans strain CA10. Biosci Biotechnol Biochem 66:897-901

Ochsner UA, Vasil ML, Alsabbagh E, Parvatiyar K, Hassett DJ (2000) Role of the Pseudomonas aeruginosa oxyR-recG operon in oxidative stress defense and DNA repair: OxyR-dependent regulation of katB-ankB, ahpB, and ahpC-ahpF. J Bacteriol 182:4533-4544

Ohno-Machado L (2014) NIH's big data to knowledge initiative and the advancement of biomedical informatics. J Am Med Inform Assoc 21:193-193

Onaca C, Kieninger M, Engesser KH, Altenbuchner J (2007) Degradation of alkyl methyl ketones by Pseudomonas veronii MEK700. J Bacteriol 189:3759-3767

Palma M, DeLuca D, Worgall S, Quadri LE (2004) Transcriptome analysis of the response of Pseudomonas aeruginosa to hydrogen peroxide. J Bacteriol 186:248-252

Patel PA, Kothari VV, Kothari CR, Faldu PR, Domadia KK, Rawal CM, Bhimani HD, Parmar NR, Nathani NM, Koringa PG, Joshi CG, Kothari RK (2014) Draft genome sequence of petroleum hydrocarbon-degrading Pseudomonas aeruginosa strain PK6, isolated from the Saurashtra region of Gujarat, India. Genome Announc 2:e00002-14

Peng RH, Xiong AS, Xue Y, Fu XY, Gao F, Zhao W, Tian YS, Yao QH (2008) Microbial biodegradation of polyaromatic hydrocarbons. FEMS Microbiol Rev 32:927-955

Ravin V, Ravin N, Casjens S, Ford ME, Hatfull GF, Hendrix RW (2000) Genomic sequence and analysis of the atypical temperate bacteriophage N15. J Mol Biol 299:53-73

Sander LC, Wise SA (2011) Polycyclic aromatic hydrocarbon structure index. NIST special publication 922. National Institute of Standards and Technology, United States department of Commerce Technology Administration, Gaithersburg

Savli H, Karadenizli A, Kolayli F, Gundes S, Ozbek U, Vahaboglu H (2003) Expression stability of six housekeeping genes: a proposal for resistance gene quantification studies of Pseudomonas aeruginosa by real-time quantitative RT-PCR. J Merd Microbiol 52:403-408

Schuster M, Hawkins AC, Harwood CS, Greenberg EP (2004) The Pseudomonas aeruginosa RpoS regulon and its relationship to quorum sensing. Mol Microbiol 51:973-985

Seo J, Keum Y, Hu Y, Lee S, Li QX (2006) Phenanthrene degradation in Arthrobacter sp. P1-1: initial 1,2-, 3,4- and 9,10-dioxygenation, and meta- and ortho-cleavages of naphthalene-1,2-diol after its formation from naphthalene-1,2-dicarboxylic acid and hydroxyl naphthoic acids. Chemosphere 65:2388-2394

Seo J, Keum Y, Hu Y, Lee S, Li QX (2007) Degradation of phenanthrene by Burkholderia sp. C3: initial 1,2- and 3,4-dioxygenation and meta- and ortho-cleavage of naphthalene-1,2-diol. Biodegradation 18:123-131

Small DA, Chang W, Toghrol F, Bentley WE (2007) Comparative global transcription analysis of sodium hypochlorite, peracetic acid, and hydrogen peroxide on Pseudomonas aeruginosa. Appl Microbiol Biotechnol 76:1093-1105

Stewart PS, Franklin MJ (2008) Physiological heterogeneity in biofilms. Nat Rev Microbiol 6:199-210

Stewart PS, Franklin MJ, Williamson KS, Folsom JP, Boegli L, James GA (2015) Contribution of stress responses to antibiotic tolerance in Pseudomonas aeruginosa biofilms. Antimicrob Agents Chemother 59:3838-3847

Wang Y, Vazquez-Duhalt R, Pickard MA (2003) Manganese-lignin peroxidase hybrid from Bjerkandera adusta oxidizes polycyclic aromatic hydrocarbons more actively in the absence of manganese. Can J Microbiol 49:675-682

Warhurst AM, Fewson CA (1994) Biotransformations catalyzed by the genus Rhodococcus. Crit Rev Biotechnol 14:29-73

Williamson KS, Richards LA, Perez-Osorio AC, Pitts B, McInnerney K, Stewart PS, Franklin MJ (2012) Heterogeneity in Pseudomonas aeruginosa biofilms includes expression of ribosome hibernation factors in the antibiotic-tolerant subpopulation and hypoxiainduced stress response in the metabolically active population. J Bacteriol 194:2062-2073

Winsor GL, Lam DK, Fleming L, Lo R, Whiteside MD, Yu NY, Hancock RE, Brinkman FS (2011) Pseudomonas genome database: improved comparative analysis and population genomics capability for Pseudomonas genomes. Nucleic Acids Res 39:D596-D600

Winsor GL, Griffiths EJ, Lo R, Dhillon BK, Shay JA, Brinkman FS (2016) Enhanced annotations and features for comparing thousands of Pseudomonas genomes in the Pseudomonas genome database. Nucleic Acids Res 44:D646-D653

Xia X, Xia N, Lai Y, Dong J, Zhao P, Zhu B, Li Z, Ye W, Yuan Y, Huang J (2015) Response of PAH-degrading genes to PAH bioavailability in the overlying water, suspended sediment, and deposited sediment of the Yangtze River. Chemosphere 128:236-244

Yan J, Wang L, Fu PP, Yu H (2004) Photomutagenicity of 16 polycyclic aromatic hydrocarbons from the US EPA priority pollutant list. Mutat Res 557:99-108

Yang Y, Chen RF, Shiaris MP (1994) Metabolism of naphthalene fluorene and phenanthrene: preliminary characterization of a cloned gene cluster from Pseudomonas putida NCIB9816. J Bacteriol 176: 2158-2164

Zhao Q, Hu H, Wang W, Peng H, Zhang X (2015) Genome sequence of Sphingobium yanoikuyae B1, a polycyclic aromatic hydrocarbondegrading strain. Genome Announc 3:e01522-14 